3 0

\title{
Carbon nanomaterial-based electrochemical biosensors for label-free sensing of environmental pollutants
}

\author{
Pankaj Ramnani ${ }^{1, \#}$, Nuvia M. Saucedo ${ }^{2, \#}$, Ashok Mulchandani1 ${ }^{1, *}$ \\ Departments of Chemical and Environmental Engineering ${ }^{1}$ and Chemistry ${ }^{2}$ \\ University of California, Riverside, CA 92521
}

\section{\# Equal contributions}

*Corresponding author: adani@engr.ucr.edu

Abstract: Carbon allotropes such as graphene and carbon nanotubes, have been incorporated in electrochemical biosensors for highly sensitive and selective detection of various analytes. The superior physical and electrical properties like high carrier mobility, ambipolar electric field effect, high surface area, flexibility and their compatibility with microfabrication techniques makes these carbon nanomaterials easy to integrate in field-effect transistor (FET)/chemiresistor type configuration which is suitable for portable and point-of-use/field-deployable sensors. This review covers the synthesis of carbon nanostructures (graphene and CNTs) and their integration into devices using various fabrication methods. Finally, we discuss the recent reports showing different sensing platforms that incorporate biomolecules like enzymes, antibodies and aptamers as recognition elements for fabrication of simple, low cost, compact biosensors that can be used for on-site, rapid environmental monitoring of environmental pollutants like pathogens, heavy metals, pesticides and explosives.

Keywords: Carbon nanotubes; graphene; electrochemistry; pollutants; aptamers; antibodies. 


\section{Contents}

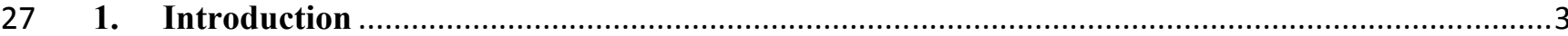

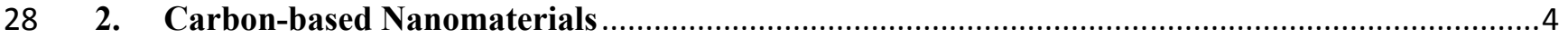

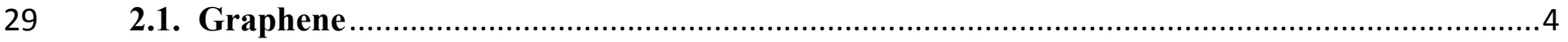

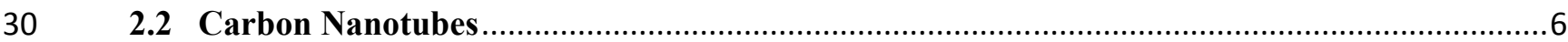

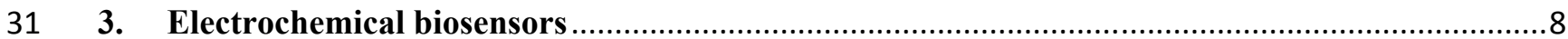

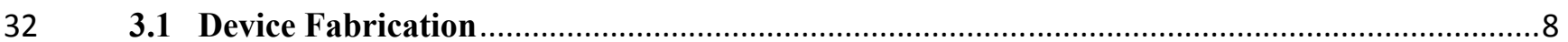

$33 \quad 3.2$ Functionalization of electrode surface with biomolecules ...................................................... 10

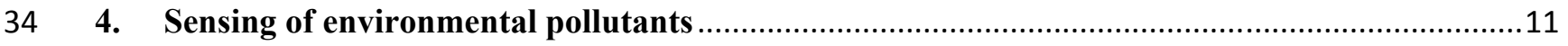

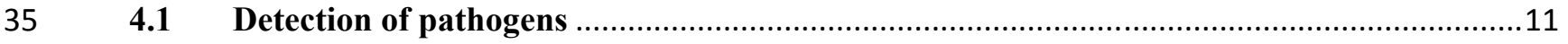

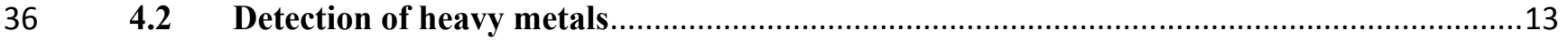

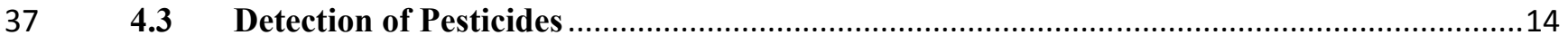

$38 \quad 4.4 \quad$ Detection of small organic molecules ….....................................................................

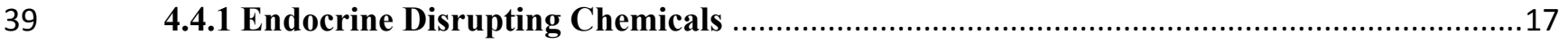

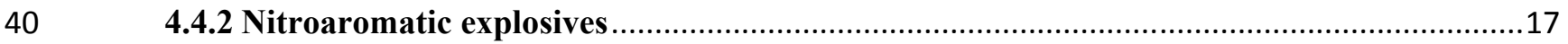

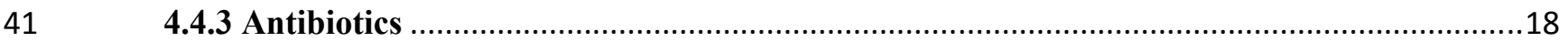

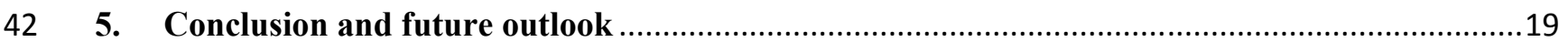

43

44 


\section{Introduction}

With the rapid industrialization and urbanization over the past few decades, environmental issues like the pollution of air, water and other natural resources have been a major global concern. Some of the major anthropogenic sources that contribute towards the deterioration of environments are derived from industrial and agricultural activities such as improper solid waste disposal and bioaccumulation of toxic wastes, massive use of pesticides and synthetic fertilizers, use of biological warfare agents, etc. Humans are often exposed to a myriad of adverse health effects due to the toxicity of these pollutants, mainly through the ingestion of contaminated food and drinking water, as well as the inhalation of ambient air with high concentrations of pollutants. These issues emphasize the importance of developing reliable, 56 highly sensitive, user-friendly and cost effective tools for constant monitoring and detection of pollutants in different mediums.

In spite of recent advances in technology, using inexpensive yet reliable devices for the selective and accurate detection of pollutants in complex samples remains a challenge due to interfering species often found within the sample matrix. The most commonly used analytical techniques for detection include gas chromatography/mass spectrometry (GC/MS) (Auroux et al., 2002) and atomic absorption spectroscopy (AAS) (Van Loon, 2012). Though highly sensitive, these analytical techniques are time-consuming, expensive, require a lot of expertise to be operated and are not easy to be deployed in the field due to their bulky size. Overcoming these limitations, chemical sensors have emerged as an alternative, a simple, rapid, cost-effective and portable tool for analyzing environmental security threats. Biosensors, a class of chemical sensors, are self-contained integrated devices that are capable of providing specific qualitative or semi-quantitative analytical information by integrating a biorecognition element which is in direct spatial contact with a transduction element (Thévenot et al., 2001). The biorecognition element, usually an enzyme, antibody or an oligonucleotide selectivity binds a specific analyte

71 from the given sample and the transducer element converts the chemical event into a measurable

72 signal like current, mass, fluorescence or $\mathrm{pH}$ change. Among these different platforms, sensors

73 based on a change in electrical property remain an attractive option due to their simple operation, 
facile fabrication process and device integration. They typically operate by measuring the change

75 in conductivity of the sensor upon adsorption of the analyte molecule onto the sensory element.

\section{Carbon-based Nanomaterials}

78

Over the past two decades, advancements in the field of nanotechnology towards synthesis of nanomaterials and understanding their fundamental properties have been substantial. Carbon-based nanostructures, including carbon nanotubes, graphene and graphene oxide, have been hot topics in the field of material science (Geim and Novoselov, 2007). Owing to their intriguing physical, chemical and electrical properties, carbon-based nanomaterials have thus emerged as potential candidates for development of next generation miniaturized biosensors (Wang, 2005; Shao et al., 2010; Zhou et al., 2009). The planar geometry of graphene and tubular geometry of carbon nanotubes ensures exposure of almost all the surface atoms which enables binding a significant fraction of analyte molecules to the transduction material. The Debye length, $\lambda_{\mathrm{D}}$, a measure of the field penetration into the bulk, is comparable to the dimensions of these nanostructures, which causes significant modulation of their electronic properties upon exposure to chemicals. These properties enable label-free detection of analytes with higher sensitivities and lower limits of detection. Furthermore, they can easily be configured as fieldeffect transistors (FETs) using lithography techniques and integrated with the modern microelectronics for fabrication of multiplexed devices that can detect a number of analytes simultaneously (Cullen et al., 1990). Apart from graphene and carbon nanotubes, other carbonbased nanomaterials, such as carbon nanofibres (CNFs) (Periyakaruppan et al., 2011; Adabi et al., 2015) and nanocrystalline diamond (NCD) (Härtl et al., 2004) also exhibit improved electrochemical activity. However, the number of reports exploring the use of these nanomaterials for electrochemical detection of environmental pollutants are limited. The scope of this review is to discuss the latest progress in the field of electrochemical biosensors based on graphene and carbon nanotubes for sensing of environmental pollutants such as pathogens, heavy metals, pesticides and small organic pollutants.

\subsection{Graphene}

\section{Structure and properties:}


Graphene is a two-dimensional (2-D) sheet of $\mathrm{sp}^{2}$ hybridized carbon atoms arranged in a honeycomb shaped lattice. As shown in Fig. 1(a), graphene is the basic building block of other carbon allotropes as it can be wrapped to get 0-D fullerenes, rolled up cylindrically to get pseudo 1-D carbon nanotubes and stacked to form 3-D graphite. (Geim and Novoselov, 2007) The twodimensional geometry of graphene results in a very high surface area (theoretically calculated to be $2630 \mathrm{~m}^{2} / \mathrm{g}$, almost twice as that of SWCNTs), thus exposing a significant fraction of surface atoms to analytes. Graphene is a semiconductor with a zero band-gap. It exhibits ambipolar electric field effect with charge carrier mobilities exceeding $15,000-20,000 \mathrm{~cm}^{2} / \mathrm{Vs}$ even at room temperature (Fig. 1(b)). This makes the electronic properties of graphene sensitive to both electron-donating and electron-withdrawing molecules. In addition, graphene exhibits superior mechanical and thermal properties, optical transparency $(\sim 97.7 \%)$ and flexibility. These physical, chemical and electrical properties make graphene an attractive candidate for the fabrication of label-free electrochemical biosensors with high sensitivity (Huang et al., 2011; Ohno et al., 2010).

\section{Synthesis:}

Exfoliation: Graphene was first isolated in 2004 at the University of Manchester by Novoselov and Geim using scotch-tape exfoliation of highly oriented pyrolytic graphite (HOPG) (Novoselov et al., 2004). This method, also called micromechanical exfoliation or peel-off method, relies on isolation of individual $\pi$-stacked layers in graphite by repeated peeling using a scotch tape. Graphene obtained using this method is usually defect-free and of high quality, however, the sample size is very small (few microns) and suffers from poor yield. This method has been limited to lab-scale fundamental studies and not for commercial synthesis of graphene. To overcome these limitations, liquid-phase exfoliation of graphite in solvents like N-methylpyrrolidone (Hernandez et al., 2008) and surfactant (sodium dodecyl benzene sulfonate) solution has been demonstrated (Lotya et al., 2009). This technique, though facile, has its own limitations such as the resulting graphene film dimensions and the incomplete removal of solvents which tend to have high boiling points. Due to its low cost and low number of processing steps involved, this technique provides a potential route for large-scale synthesis of graphene-based sensors. 
Reduced graphene oxide: Graphite oxide (GO) is synthesized by Hummers method which involves treatment with potassium permanganate $\left(\mathrm{KMnO}_{4}\right)$ and concentrated sulfuric acid $\left(\mathrm{H}_{2} \mathrm{SO}_{4}\right)$ for simultaneous oxidation and exfoliation of graphite (Hummers and Offeman, 1958). The acid treatment introduces polar oxygen functional groups like epoxies, carbonyls, hydroxyls, etc. which makes GO hydrophilic. Using mild sonication, the GO sheets can be dispersed with ease in many solvents, particularly well in water (Paredes et al., 2008). Thereafter, the colloidal solution of graphene oxide can be reduced to graphene using different treatments like chemical reduction by directly adding reducing agents like hydrazine (Stankovich et al., 2007) or by thermal reduction using elevated temperatures $\left(200-900{ }^{\circ} \mathrm{C}\right)$ (McAllister et al., 2007). Electrochemical reduction by immobilizing the GO onto an electrode surface and successively performing reducing scans by sweeping potential from 0 to $-1.5 \mathrm{~V}$ is perhaps the simplest and most convenient method to obtain reduced graphene oxide (rGO) (Zhou et al., 2009) ready for use as transducer. Typical dimensions of rGO flakes thus obtained vary from sub-micron to few microns. The reduced graphene oxide still contains some defect sites in the form of $\mathrm{sp}^{3}$ centers and oxygen functional groups due to incomplete reduction. These functional groups can be used for direct covalent binding of biological receptor and are known to enhance the electrocatalytic properties of graphene.

Chemical vapor deposition (CVD): The fabrication of graphene-modified electrodes and graphene-FET devices for sensing applications requires bulk production of large-area graphene films. Chemical vapor deposition on transition metal substrates like Ni (Obraztsov et al., 2007), Pd (Hamilton and Blakely, 1980), Pt (Zi-Pu et al., 1987) and Cu (Mattevi et al., 2011) is a promising, cost-effective and high-throughput method for synthesizing large-area graphene films with relatively fewer defects. CVD synthesis of graphene, simply put, is the subsequent diffusion and surface segregation of carbon atoms, obtained during the thermal decomposition of introduced hydrocarbons, upon cooling of the carbon-metal solid solution (Fig. 2). In particular, CVD synthesis of graphene on $\mathrm{Cu}$ has gathered the most interest due to low solubility of carbon in copper. This method gives control over the grain size, crystallinity and number of graphene layers by regulating the gas flow rate, growth time and cooling rate. The defect-free transfer of graphene onto desired substrates has also been studied in depth (Srivastava et al., 2010). This makes integration of graphene with conventional Si-based electronics much easier and serves as a platform for fabrication of miniaturized biosensors. 


\subsection{Carbon Nanotubes}

167

168

169

170

171

172

173

174

175

176

177

178

179

180

181

182

183

184

185

186

187

188

189

190

191

192

193

194

195

\section{Structure and properties:}

Carbon nanotubes can be visualized as cylindrical tubes 'rolled' up from single or multiple sheets of graphene containing $\mathrm{sp}^{2}$ hybridized carbon atoms arranged in honeycomb lattice. CNTs can be classified as either single-walled carbon nanotubes (SWNTs) or multi-walled carbon nanotubes (MWNTs). SWNTs consist of a single sheet of graphene seamlessly wrapped into a cylindrical tube. They are approximately 0.4 to $2 \mathrm{~nm}$ in diameter and 1 to $100 \mu \mathrm{m}$ long depending upon the synthesis method used while MWNT consist of concentric cylinders of rolled up graphene sheets. The diameter of MWNTs is between 1-10 nm depending on the number of graphene sheets. The tube walls of MWNTs are parallel to a central axis and the interlayer spacing between the tubes is about $0.34 \mathrm{~nm}$. SWNTs can be of metallic or semiconducting nature depending on their chirality and the diameter of the tubes (Wilder et al., 1998). The chirality can be designated as armchair, zigzag or chiral depending on the axis upon which the CNT was rolled (Fig 3). Armchair SWNTs are metallic in nature (zero band-gap) and therefore cannot be used for chemiresistive/FET based sensor fabrication which requires semi-conducting material. SWNTs of other chiralities are semi-conducting in nature with band-gap depending primarily on tube diameter (Odom et al., 1998; Dresselhaus et al., 2004).

\section{Synthesis:}

There are three primary methods of CNTs synthesis: arc discharge (Iijima, 1991), chemical vapor deposition (CVD) (Kong et al., 1998), and laser ablation (Guo et al., 1995). CNTs were first obtained using carbon-arc discharge. Depending upon the catalyst used, this method yields MWNTs or SWNTs with high yield $(\sim 90 \%)$ and gives better control over the dimensions of synthesized tubes (diameter between 0.6-1.4 nm for SWNTs and about $10 \mathrm{~nm}$ for MWNTs and tube length close to few microns). The high yield and reproducibility make this method suitable for large-scale commercial synthesis. Chemical vapor deposition, much like arc discharge, is also catalyst dependent and results in small diameter CNTs. Though the yield of CNTs is comparatively lower, the resulting CNTs are much cleaner needing less cleanup of soot and crude products. Laser ablation gives the cleanest CNT though at a higher price and lower yield with SWNT diameters of 1-2 $\mathrm{nm}$. All these synthesis methods result in a mixture of metallic and 
semi-conducting CNTs. Now, issues that still remain to be addressed are: 1) separation of CNTs from impurities which usually require harsh treatments that often damage CNTs resulting in shortened length or defected CNT surfaces, 2) the separation of metallic CNTs from semiconductive CNTs and 3) reducing the formation of CNT bundles which occurs naturally due to van der Waals attractions. CNT cleanup approaches can vary depending on the initial method of synthesis. Separation of metallic and semi-conductive CNTs can be done through selective functionalization (Maeda et al., 2008), selective destruction by electrical heating (Collins et al., 2001), or separation by density gradient ultracentrifugation (Lipscomb et al., 2011), among others (Scheibe et al., 2011). To decrease bundle formation, CNTs can be functionalized with chemical groups or suspended in a surfactant containing solution (Guldi et al., 2003).

\section{Electrochemical biosensors}

As discussed earlier, the geometry of carbon nanomaterials, 2-D (planar) in case of graphene and 1-D (tubular) for CNTs, makes them ideal transduction materials. Additionally, their electrochemical properties such as faster electron transfer kinetics, low residual current, readily renewable surface, wide potential window and enhanced currents with significant reduction in over-potential make carbon nanomaterials promising candidates for biosensing applications in comparison to other carbon-based electrode materials like glassy carbon (Wang et al., 2009). It has been experimentally demonstrated that the electrochemistry of graphene is driven by the edge-plane sites that can exhibit an electron transfer rate $\left(\mathrm{k}_{\mathrm{e}}\right)$ as high as $0.01 \mathrm{~cm} / \mathrm{s}$ (Pumera et al., 2010). So, the high density of edge-plane defect sites per unit mass of the material in carbon nanotubes and chemically modified graphene enhances their electrochemical activity, making them highly sensitive and selective transduction materials in comparison to other nanomaterials such as metal oxide nanowires and conducting polymer films (Kong et al., 2000).

\subsection{Device Fabrication}

Electrochemical biosensors based on graphene and CNTs can broadly be divided into two device architectures: (i) conventional three electrode set-up, (ii) chemiresistive/FET set-up (Balasubramanian and Burghard, 2006).

The three electrode set-up, commonly used for amperometric, impedimetric and potentiometric biosensors comprises of a working (WE), reference (RE) and counter-electrode 
227 (CE). Fig. 4 shows the schematic representation of a conventional three electrode system for electrochemical detection of analytes. For working electrodes, the commonly used glassy carbon electrode (GCE) surface is modified by depositing a film of graphene or carbon nanotubes. Graphene flakes obtained by exfoliation and dispersed in surfactant solution are drop-casted onto the bare GCE surface, baked at slightly elevated temperatures $\left(40-60^{\circ} \mathrm{C}\right)$ and washed repeatedly with deionized water. In the case of carbon nanotubes, due to their low solubility in most solvents, CNT-modified electrodes are prepared by casting of CNT/sulfuric acid solution on GCE surface. Nafion, a perfluorosulfonated polymer, is also used extensively to solubilize CNTs (Wang et al., 2003). The resulting biosensors greatly benefit from the coupling of the unique ionexchange and antifouling properties of Nafion films with the efficient electrocatalytic properties of CNTs.

For the fabrication of chemiresistive/field-effect transistor (FET)-type biosensors, graphene is first transferred on to a substrate due to its planar geometry, followed by patterning of metal contacts using electron beam lithography or photolithography (He et al., 2012). On the other hand, carbon nanotube-based FETs are typically fabricated by first patterning the metal electrodes on the substrate followed by bridging of the electrode gap with carbon nanotubes (Kim et al., 2007). Fig. 5(a,b) shows the typical configuration of a FET-type biosensor with the carbon nanomaterial (graphene or CNTs) as a channel connecting the source (S) and drain (D) electrodes. The current-voltage $(I-V)$ characteristics between the source and drain for various gate voltages or changes in current at fixed gate potential can be measured and correlated to analyte concentration. Unlike the conventional metal-oxide semiconductor field-effect transistors (MOSFETs), where the conducting channel is buried in the bulk material, the nanotubes or graphene are located on the surface of the supporting substrate in direct contact with the environment. (Liu and Guo, 2012; Xu et al., 2014) Upon incubation and specific binding of chemical/biological analytes, the conductance changes and the change is used to quantify the analyte concentration. An alternate transistor configuration shown in Fig. 5(c, d) with a twoterminal electrodes and no gate, called a chemiresistor, can also be used. In a chemiresistor, the 255 256 absorbed molecules on the surface are used to modulate the conductance instead of a gate electrode (Kim et al., 2007).

The planar geometry of graphene makes it easier to deposit on the flat substrate. CVDgrown, large-area graphene films are deposited using a polymer (PMMA) support film and the 
polymer film is eventually dissolved in acetone. For anchoring carbon nanotubes, different methods have been discussed in literature including direct growth on substrate using chemical vapor deposition (CVD) growth (Byon and Choi, 2006), drop casting (DC) (Zheng et al., 2003), and dielectrophoresis (DEP) (Tlili et al., 2010). Dielectrophoretic alignment methods prevent the bundling of CNTs and aligns individual CNTs across the source-drain electrodes. A modified drop casting method has been demonstrated where the $\mathrm{Si} / \mathrm{SiO}_{2}$ wafer is treated with APTES (3aminopropyltriethoxysilane) which leads to a monolayer of aminosilane, a chemical known for its affinity to CNTs. Incubation with dilute SWNT solution leads to a uniformly distributed highdensity network of SWNTs (Sarkar et al., 2013). The process can be used for wafer level fabrication of CNT sensors. The techniques of DC, DEP and APTES-assisted self-assembly have been used for making rGO-based chemiresistor/FET devices (Becerril et al., 2008; An et al., 2010).

\subsection{Functionalization of electrode surface with biomolecules}

For the detection of analytes from complex matrices, the transducer element of the biosensor has to be coupled with a biorecognition molecule, i.e. enzymes, antibodies, aptamers, etc., for enhancing the biosensor selectivity and achieving high sensitivities without using labeling or amplification techniques. The two general approaches for modification of the electrode surface with biomolecules are: covalent and non-covalent functionalization. For covalent attachment of biomolecules, carbon nanotubes and graphene are oxidized to introduce carboxylic (-COOH) groups on the sidewalls and edge-planes of carbon nanotubes and graphene (Datsyuk et al., 2008); reduced graphene oxide already has $-\mathrm{COOH}$ groups. These carboxylic groups couple with the amine $\left(-\mathrm{NH}_{2}\right)$ groups in protein or the amine terminated ends of an aptamer via amide bond formation. Though effective, covalent modifications disrupt the in-plane $\mathrm{sp}^{2}$ bonding in the carbon lattice pattern and introduce defects that significantly affect the desirable electrical properties of the nanomaterials (Banerjee et al., 2005). Non-covalent functionalization of carbon nanomaterials, on the other hand, modifies the electrode surface in a non-destructive manner and also enhances the solution phase stability of the nanomaterials by preventing restacking or aggregation. This can be achieved by either direct physical adsorption of the biomolecules on the surface of the SWNT/graphene or using a bifunctional linker molecule that can be grafted to the sidewalls of CNTs or planar graphene (Chen et al., 2001). An 
example of such bilinker is 1-pyrenebutanoic acid succinimidyl ester (PBASE) in which the pyrene binds to SWNT non-covalently through $\pi-\pi$ interaction and the succinimidyl ester forms an amide bond with the amine group of a biomolecule (Fig. 6). In order to prevent the nonspecific binding of the biomolecules, the uncoated areas of graphene/CNT can be coated with a surfactant such as Tween 20 or using an inert protein bovine serum albumin (BSA) (Steinitz, 2000).

\section{Sensing of environmental pollutants}

\subsection{Detection of pathogens}

Microbial diseases due to infections from bacteria, viruses and other pathogenic microorganisms constitute the major cause of death in many developing countries. These pathogens could and have been used as biological warfare agents. Hence, rapid and cost-effective detection of pathogens is essential for proper diagnosis, outbreak prevention and prescribing correct therapeutic treatment. The commonly used methods for detection like polymerase chain reaction (PCR) (Rodríguez-Lázaro et al., 2005) and enzyme-linked immunosorbent assay ELISA (Crowther, 1995), though sensitive and selective, are multi-step and additionally require sample pretreatment and long assay times. Carbon nanomaterial-based electrochemical biosensors, owing to their high sensitivity and specificity, facilitate detection of a broad spectrum of analytes in complex sample matrices and do so in a simple and cost-effective manner.

By functionalizing carbon nanomaterials with antibodies specific to the pathogen of interest, highly selective and sensitive detection of bacteria and viruses can be achieved. Liu et al. (Liu et al., 2013) reported a reduced graphene oxide - FET biosensor targeting viruses using antibodies (see Fig. 7). Rotavirus, a water-borne virus that is the leading cause of severe diarrhea among infants and young children, was used as a model virus. Single-layered and largearea graphene oxide sheets with grain sizes greater than $50 \mu \mathrm{m}$ were spin-coated on a $\mathrm{Si} / \mathrm{SiO}_{2}$ substrate and thermally reduced to reduced graphene oxide (rGO). The electrodes were patterned using photolithography and a PDMS microfluidic channel was bonded to the substrate. Reduced graphene oxide was then non-covalently functionalized with rotavirus-specific antibody using PBASE as a linker molecule. Solutions containing different concentrations of rotavirus were loaded and the virus captured by the antibody was monitored in real time by measuring the 
current changes. For analysis of real samples, goose feces spiked with rotavirus were used for detection. The limit of detection was reported to be $10^{2} \mathrm{pfu} / \mathrm{mL}$. Similarly, we demonstrated highly sensitive and selective detection of E. coli O157:H7 and T7 bacteriophage using SWNTbased chemiresistive sensors functionalized with respective antibodies (Garcia-Aljaro et al., 2010).

Apart from using antibodies, short DNA sequences called aptamers have been used as highly specific bioreceptors for high proficiency detection of a wide range of analytes including bacteria. Zelada-Guillén et al. (2009) used aptamer-functionalized CNTs for selective detection of Salmonella Typhi from a complex mixture. SWNTs were carboxylated via acid treatment and sprayed onto a $3 \mathrm{~mm}$ diameter glassy carbon electrode (GCE) to achieve a $30 \mu \mathrm{m}$-thick SWNT network. The aptamer was modified with a five-carbon spacer and an amine group ($\left.\left(\mathrm{CH}_{2}\right)_{5} \mathrm{NH}_{2}\right)$ at the $3^{\prime}$ end and was covalently bound to the carboxylic groups on the sidewalls of SWNTs using carbodiimide chemistry. Upon introduction of the target bacteria, a conformational change in the aptamer is induced due to separation of the phosphate groups from the SWNT surface. This changes the charge density near the electrode surface and a subsequent change in potential is recorded between the SWNT-aptamer modified GCE (working electrode) and $\mathrm{Ag} / \mathrm{AgCl}$ electrode (reference). The potentiometric response of the biosensor was measured for bacteria concentrations varying from $0.2 \mathrm{cfu} / \mathrm{ml}(1 \mathrm{CFU}$ in $5 \mathrm{ml} \mathrm{PB})$ to $10^{6} \mathrm{cfu} / \mathrm{mL}$. As shown in Fig. 8, upon increasing concentration of bacteria, the potential difference increased but the magnitude of signal decreased, possibly due to the saturation of binding sites. The average response was reported to be $1.87 \mathrm{mV}$ per $\log$ of bacteria concentration. The specificity of the sensor was demonstrated by testing against non-target bacteria (Escherichia coli and Lactobacillus casei). Similarly, So et al. (2008) reported an aptamer-functionalized SWNT-based FET for selective and sensitive detection of $E$. coli.

Huang et al. (2011) demonstrated a graphene-based biosensor that can detect the metabolic activities of live bacterial cells in real time. Such simple, rapid and label-free biosensors demonstrate the ability to distinguish live and dead cells and can be used for high throughput screening of antibacterial drugs. CVD grown graphene was functionalized with a linker molecule and incubated with anti-E. coli $\mathrm{O}$ and $\mathrm{K}$ antibody. The device was incubated with ethanolamine and Tween 20 to passivate the unreacted linker molecules and uncoated graphene, respectively. Different concentrations of $E$. coli solution were added to the device. The 
attachment of bacteria by antibodies was monitored by taking $I_{d^{-}}-V_{d s}$ and FET transfer curves $\left(I_{d^{-}}\right.$ $\mathrm{V}_{\mathrm{g}}$ ) before and after each incubation of $E$. coli bacteria from $0-10^{5} \mathrm{cfu} / \mathrm{mL}$ (Fig. 9). Incubation of device with Pseudomonas aeruginosa did not produce a significant change in response of FET, indicating the high specificity of detection. To evaluate and confirm device $\mathrm{pH}$ sensitivity, the device was incubated with different $\mathrm{pH}$ buffer solutions ( $\mathrm{pH} 3-11$, odd only) and three different measurements were taken: $I_{d}-V_{d s}, I_{d}-V_{g}$, and real-time current (shown in Fig. 10(a)). For the detection of live bacterial cells, real-time current was recorded for devices incubated with $10^{5} \mathrm{cfu} / \mathrm{mL}$ E. coli bacteria. The addition of glucose into the phosphate buffer saline hydrated device induced metabolization and subsequent release of acidic metabolites (e.g. pyruvic, citric, and lactic acid) from the living bacterial cells, thus changing the $\mathrm{pH}$ near the electrode and causing a drop in the current (Fig. 10(b)). An LOD of $10 \mathrm{cfu} / \mathrm{mL}$ was achieved for the capture of E. coli bacteria, while LOD for live bacteria was not explored.

The reports discussed above successfully demonstrate that CNTs and graphene coupled with a variety of recognition elements can be used for highly sensitive and selective sensing of pathogens from environmental monitoring and diagnosis viewpoints.

\subsection{Detection of heavy metals}

Heavy metals are considered one of the major pollutants of the biosphere due to their toxicity and lengthy persistence in the environment. Sources of wastewater contain heavy metals including lead $(\mathrm{Pb})$, cadmium $(\mathrm{Cd})$, mercury $(\mathrm{Hg})$, copper $(\mathrm{Cu})$, zinc $(\mathrm{Zn})$, nickel $(\mathrm{Ni})$, etc., mostly from metallurgical industries such as mining, smelting, electroplating, automotive, battery and electric cable manufacturing (Yilmaz et al., 2007). Therefore, an acute need exists for the detection and real-time monitoring of these heavy metals in natural and industrial environments.

The current detection methods based on spectroscopy (AAS, AES, ICP-MS, etc.; Burlingame et al., 1996; Jackson et al., 1996) are expensive and not suitable for on-site detection. Additionally, they can only detect the total amount of heavy metal and not the bioavailable concentrations accessible to the living organisms. Enzyme-based biosensors have been reported in literature for highly sensitive detection of heavy metals. The detection scheme is based on the inhibition of enzyme activity upon conjugation of metal ions with thiol groups in the enzyme (Wanekaya et al., 2008). But these biosensors lack selectivity due to interference from other 
heavy metal ions and pesticides present in aqueous environments. Furthermore, due to the poor stability of enzymes at physiological pH, enzyme-based biosensors are not suitable for practical real-time detection of heavy metals.

DNA-functionalized CNT biosensors (aptasensors) have become a widely used tool to study the interactions of DNA with many heavy metal ions due to the stability of DNA in physiological pH environments. Liu and Wei (2008) reported an electrochemical biosensor based on DNA-functionalized, single-walled carbon nanotube (DNA-SWNT) hybrid-modified glassy carbon electrode (GCE) for the detection of Arsenic (As) which is documented as a toxic mineral and an anti-cancer drug. The detection was achieved by directly detecting the current resulting from oxidation of $\operatorname{As}(0)$ on the biosensor surface to $\mathrm{As}(\mathrm{III})$ during the anodic stripping step. The biosensor showed a limit of detection of $0.05 \mu \mathrm{g} / \mathrm{L}$ at $\mathrm{pH} 7.0$ without significant loss of sensitivity over multiple runs.

Gong et al. (2013) proposed a label-free, chemiresistive biosensor for $\mathrm{Hg}^{2+}$ ion detection based on polyT:polyA duplex-functionalized SWNTs. The detection scheme is shown in Fig. 11. In brief, the SWNT chemiresistive biosensor was fabricated through non-covalent functionalization of SWNTs with a linker molecule (PBASE) via $\pi$ - $\pi$ bond interaction. The amino-labeled oligonucleotide polyT (5'-/5AmMC6/TTT TTT TTT TTT TTT-3') was covalently attached to the linker via amide bond formation followed by hybridization with polyA to form a polyT:polyA duplex. Upon incubation with $\mathrm{Hg}^{2+}$ solution, the resistance/conductance of the SWNT chemiresistor device changed due to the dehybridization of polyT:polyA duplex which occurs simultaneously with the formation of $\mathrm{T}-\mathrm{Hg}^{2+}-\mathrm{T}$ duplex and the release of polyA from the SWNTs surface. As shown in Fig. 12(a), the calibration curve with the normalized change in resistance for varying concentrations of $\mathrm{Hg}^{2+}$ showed a linear response for $\mathrm{Hg}^{2+}$ concentrations ranging from $100 \mathrm{nM}$ to $1 \mu \mathrm{M}$ with a $6.72 \times 10^{-3} \mathrm{nM}^{-1}$ sensitivity. The selectivity of the biosensors was tested by measuring the response upon incubation with different metal ion solutions (Fig. 12(b)).

Lian et al. (2014) proposed a novel electrochemical sensor with DNA wrapped metallicSWNTs for detection of $\mathrm{Pb}^{2+}$ in water. The mechanism of detection, shown in Fig. 13, was based on the ability of $\mathrm{Pb}^{2+}$ to form complexes with DNA by binding to negatively charged phosphate groups (Liu et al., 2009) or to the bases of DNA structures (Oliveira et al., 2008). The differential pulse voltammetry response showed a linear relation between the peak currents and $\mathrm{Pb}^{2+}$ 
412 concentration and a detection limit of $0.41 \mathrm{nM}$ in buffer solution was established. The high 413 selectivity and anti-interference ability of the sensor was demonstrated by examining the 414 influence of foreign species like $\mathrm{Cu}^{2+}, \mathrm{Ca}^{2+}, \mathrm{Al}^{3+}, \mathrm{Ni}^{2+}$ and $\mathrm{Co}^{2+}$. Finally, the sensor was used to

415 determine $\mathrm{Pb}^{2+}$ concentrations in river and tap water and the results showed good agreement with 416 standard methods like atomic absorption spectrometer (AAS), thus showing the reliability and 417 accuracy of the proposed sensor.

\subsection{Detection of Pesticides}

Pesticides are extensively used in agricultural activities to control certain forms of plant or animal life and increase the production yield. However pesticides are well known toxic pollutants that are hazardous to human health. Organophosphates (OPs), the most commonly used toxic substance in pesticides, can lead to cholinergic dysfunction, which affects the health of both humans and animals. Rapid, sensitive and cost-effective detection of OP neurotoxins is important for protecting water resources and food supplies, in the defense against terrorist activity, and for monitoring detoxification processes. Traditional laboratory-based analytical techniques for detecting OPs include chromatography and mass spectrometry which are rather time-consuming and expensive (Sherma, 1993). Enzyme-based biosensors using carbon nanotubes as the transducer have been used for highly selective and rapid detection of pesticides. These enzymatic biosensors can either be used for direct detection based on the catalytic activity of organophosphorus hydrolase (OPH) (Mulchandani et al., 2001) or based on the inhibition of activity of several enzymes present in pesticides like acetylcholinesterase (AChE), butyrylcholinesterase (BChE) and tyrosinase (Tyr) (Andreescu and Marty, 2006 and Tanimoto de Albuquerque and Ferreira, 2007). prevents nerve transmission by blocking breakdown of the transmitter choline. Biosensors based on the inhibition to $\mathrm{AChE}$ have been widely used for the detection of OP and carbamate pesticides. Du et al. (2007) reported an AChE biosensor for rapid determination of triazophos, an organophophorus insecticide. A multiwalled carbon nanotube (MWNT) - chitosan composite was used as the working electrode and $\mathrm{AChE}$ was non-covalently immobilized on the electrode surface using glutaraldehyde as the cross-linker. The interaction of AChE with acetylthiocholine produced thiocholine and electro-active product which produces an irreversible oxidation peak. 
442 The inhibition effect of OP on AChE was determined by monitoring the decline of oxidation current of thiocholine for increasing concentrations of triazophos. (Fig. 14)

Although sensitive, biosensors based on AChE inhibition display poor selectivity since AChE is inhibited by neurotoxins which include not only OP pesticides but also carbamate pesticides. Since the inhibition of enzyme activity is irreversible, they are only suitable for single-time use. Also, the protocols involve multiple steps which is undesirable for detection on a real-time basis. A direct and real-time biosensing route for detection of OP neurotoxins involves the biocatalytic activity of organophophorus hydrolase $(\mathrm{OPH})$, an enzyme that has a broad substrate specificity and is able to hydrolyze a number of organic phosphorus (OP) pesticides such as paraoxon, parathion, coumaphos, diazinon, dursban, etc (Mulchandani et al., 2001). Liu et al. (2007) showed real-time detection of paraoxon, an OP pesticide, using dielectrophoretically aligned SWNTs across electrode pairs. The use of aligned carbon nanotubes ensures uniform coverage and higher interfacial contact between enzyme OPH and the SWNTs that can lead to higher sensitivity. But the non-specific binding (NSB) of the enzyme prevents the practical utility of the biosensor due to possible leaching of enzyme under operational conditions. Pedrosa et al. (2010) fabricated a biosensor by covalent immobilization of organophosphorus hydrolase enzyme on oxidized carbon nanotubes. Briefly, the CNTs (SWNTs and MWNTs separately) were oxidized by acid treatment to introduce carboxylic functional groups and the OPH enzyme was covalently immobilized by two different methods: (a) directly coupling of ethylenediamene with the carboxyl groups to introduce amino groups via amide formation (EDC-NHS), (b) direct coupling of the carboxyl groups to amine group modified with glutaraldehyde (APTES-GA). On comparing the catalytic activity of the enzyme, OPH conjugation through EDC-NHS chemistry displayed slightly better activity compared to APTES-GA chemistry. Additionally, OPH conjugated to SWNTs demonstrated much higher activity compared to OPH conjugated to MWNTs which can be explained by a more uniform SWNTs network and subsequently uniform enzyme deposition. The hydrolysis of the OP pesticide, in this case paraoxon, generates pnitrophenol which is electroactive and can be electrochemically oxidized at the anode surface. The oxidation was monitored amperometrically in real time under flow-injection (FIA) mode. As shown in Fig. 15, the oxidation current increases linearly for increasing concentrations of paraoxon for both SWNT-OPH and MWNT-OPH immobilized by the EDC-NHS process. The biosensor displayed high sensitivity and good stability with a dynamic concentration range of 
$4730.5-8.5 \mu \mathrm{mol} / \mathrm{L}$ for SWNT-based devices and a detection limit of $0.01 \mu \mathrm{mol} / \mathrm{L}$, which were attributed to the covalent functionalization of CNTs with $\mathrm{OPH}$ enzyme.

We reported a highly selective and sensitive amperomteric biosensor based on mutant of $\mathrm{OPH}$ enzyme with improved catalytic activity for phosphothiolester (organophosphorus pesticides with P-S bond) such as s-demeton (also a simulant of VX and VX-R nerve gases/chemical warfare agents). The enzyme-electrode had a dynamic linear range up to $85 \mathrm{M}$, a limit of detection of $1 \mathrm{mM}$ (equivalent to $258 \mathrm{ppb}$ ) and no interference even from other organophosphorus family pesticides such as malathion, methyl parathion, diazinon, etc. and carbamate family pesticides such as sevin and sutan (Joshi et al., 2006).

\subsection{Detection of small organic molecules}

Detection of small organic molecules like toxins, explosives, pharmaceuticals and personal care products, etc., is important from an environmental monitoring point of view. Sensing based on traditional modes of affinity sensing is challenging with methods often requiring sample concentration and signal enhancement due to their small size. However, these organic molecules of interest typically contain electroactive functional groups which can be oxidized/reduced by applying a potential. Thus, electrochemical biosensors are ideal candidates for the monitoring of these analytes in the environment.

\subsubsection{Endocrine Disrupting Chemicals}

Endocrine-disrupting chemicals (EDCs) are substances that mimic natural hormones in the endocrine system, thus causing adverse effects on human health, most notably in children and fetuses (Diamanti-Kandarakis et al., 2009). Bisphenol A (2,2-bis (4-hydroxyphenyl) propane (BPA)) is one such endocrine disruptor that is widely used as a monomer in the production of epoxy resins and polycarbonate plastics that are used for the manufacture of baby bottles, food packaging material and even medical and dental fillings.

Jiang et al. (2013) demonstrated electrochemical detection of leached BPA in plastic baby bottles by monitoring the oxidation current of BPA on glassy carbon electrode (GCE) modified with calf thymus DNA-functionalized SWNTs/Nafion composite. The electrode was prepared by drop casting of CNTs/Nafion solution onto the GCE surface and the dsDNA (1 $\mathrm{mg} / \mathrm{mL}$ ) was immobilized electrochemically by applying a fixed potential of $0.5 \mathrm{~V}$ for $300 \mathrm{~s}$. Parameters such as concentration of dsDNA, voltage scan rate and $\mathrm{pH}$ were optimized 
systematically. Fig. 16 shows the differential pulse voltammograms (DPVs) collected for different concentrations of BPA in phosphate buffer solution $(\mathrm{pH} 8.5)$ and the corresponding calibration curve. The peak at $0.51 \mathrm{~V}$ corresponds to oxidation of BPA. The biosensor showed a linear response for BPA concentrations ranging from $10 \mathrm{nM}$ to $20 \mu \mathrm{M}$ and the limit of detection was found to be $5 \mathrm{nM}$. The sensor displayed high selectivity without significant loss of activity or fouling in presence of common interferents like acetate, phosphate, chloride, metal ions and other phenolic compounds.

\subsubsection{Nitroaromatic explosives}

2,4,6-Trinitrotoluene (TNT) has a wide range of uses from explosives and ammunitions to manufacturing of dyes, plasticizers and pesticides. Traces of TNT can be found in soil and groundwater due to its persistent nature (Pennington and Brannon, 2002). Park et al. (2010) reported a carbon nanotube immunosensor/affinity biosensor for on-site, rapid and highly sensitive detection of TNT. Briefly, the SWNTs, dielectrophoretically aligned across the conducting channel, were non-covalently functionalized with trinitrophenyl-ovalbumin (TNPOVA) and ligated with an anti-TNT single chain antibody (scAb). Upon incubation of the device with TNT, which has a higher binding affinity to anti-TNT scAb, displacement of the attached $\mathrm{scAb}$ resulted and produced a change in the conductance of the device. The detection scheme is showed in Fig. 17(a). The sensor was able to detect TNT concentrations ranging from $0.5-5000$ ppb in buffer solution and real water samples spiked with TNT and displayed high selectivity in the presence of other nitroaromatic explosives (Fig. 17(b)).

\subsubsection{Antibiotics}

Aside from industrial pollutants, commercial livestock farming and household wastewater have both gained attention for their contribution to increased amounts of antibiotics in source waters (Lindsey et al., 2001). Tetracycline is a persistent antibiotic that is often detected even after wastewater treatments. Zhou et al. (2012) showed detection of tetracycline using an aptamer-MWNT-modified GCE. First, the MWNT were carboxylated via acid treatment and then drop-casted onto the glassy carbon electrode. The carboxyl groups were activated using carbodiimide chemistry after which it was incubated with amine-modified antiTET aptamer (76-base sequence) for $3 \mathrm{~h}$. As shown in Fig. 18, differential pulse voltammograms spanning from 0.05 to $0.4 \mathrm{~V}$ were collected before incubation of working electrode with 
tetracycline-containing solutions. Ferricyanide solution was used as a mediator and the oxidation peak current at $0.225 \mathrm{~V}$ was monitored to quantify the TET concentration. Incubation with tetracycline, led to an anti-TET aptamer/TET complex that hindered electron transfer and decreased the anodic peak current. As the working electrode was incubated with increasing concentrations of tetracycline, the anodic current decreased. A linear correlation was established between signal and $\log$ of concentration from $10 \mathrm{nM}$ to $100 \mu \mathrm{M}$ with an LOD of $5 \mathrm{nM}$. Specificity of sensor was demonstrated using structurally similar compounds. When testing spiked milk samples, sensor showed good recovery (88-96\%).

\section{Conclusion and future outlook}

From the reports discussed in the review employing different detection schemes, it is clear carbon nanomaterial-based biosensors offer many advantages for the detection of pollutants in natural environment. Due to outstanding physical and electrical properties of graphene and carbon nanotubes, highly selective and sensitive detection of analytes has been shown without the need of labeling or amplification techniques. Additionally, owing to the low cost of manufacturing, compact size, robustness and require low power consumption, these carbonbased nanobiosensors are suitable for field-deployable/point-of-use, rapid and multi-analyte environmental monitoring.

However, the properties of carbon nanotubes depends on a variety of factors like SWNTs/MWNTs, metallic/semi-conducting nature, length and diameter of tubes, level of agglomeration, etc. Similarly in case of graphene, the electrical properties are sensitive to factors like number of layers, impurities, edge structure, substrate, etc. Hence, the performance of the biosensor varies from device to device. But with advancements in the field of material synthesis and standardization of microfabrication protocols, fabrication of biosensors showing consistent and reproducible performance is necessary. Additionally, device integration of the detection platforms into actual biosensing systems is still in the preliminary stages and efforts are necessary for fabrication of easy-to-use platforms (example: lateral flow assays) where sample pre-treatment and incubation steps are integrated on the same platform where detection of analyte occurs. And finally, it should be mentioned that considerable work is yet to be done to assess the biocompatibility of carbon nanomaterials and to determine any possible toxicity they might have during long-term use. 
566 Acknowledgements

567 This work was supported by the National Science Foundation grants 1307671 and 1143635 , 568 USDA grant 2014-67021-21589 and the W. Ruel Johnson Chair in Environmental Engineering. 569 


\section{References}

571

572

573

574

575

576

577

578

579

580

581

582

583

584

585

586

587

588

589

590

591

592

593

594

595

596

597

598

599

600

601

602

603

604

605

Adabi, M., Saber, R., Faridi-Majidi, R., \& Faridbod, F. (2015). Performance of electrodes synthesized with polyacrylonitrile-based carbon nanofibers for application in electrochemical sensors and biosensors. Materials Science and Engineering: C, 48, 673-678.

An, S. J., Zhu, Y., Lee, S. H., Stoller, M. D., Emilsson, T., Park, S., Velamakanni, A., An, J., \& Ruoff, R. S. (2010). Thin film fabrication and simultaneous anodic reduction of deposited graphene oxide platelets by electrophoretic deposition. The Journal of Physical Chemistry Letters, 1(8), 1259-1263.

Andreescu, S., \& Marty, J. L. (2006). Twenty years research in cholinesterase biosensors: from basic research to practical applications. Biomolecular engineering, 23(1), 1-15.

Auroux, P. A., Iossifidis, D., Reyes, D. R., \& Manz, A. (2002). Micro total analysis systems. 2. Analytical standard operations and applications.Analytical chemistry, 74(12), 2637-2652.

Balasubramanian, K., \& Burghard, M. (2006). Biosensors based on carbon nanotubes. Analytical and bioanalytical chemistry, 385(3), 452-468.

Banerjee, S., Hemraj-Benny, T., \& Wong, S. S. (2005). Covalent surface chemistry of single-walled carbon nanotubes. Advanced Materials, 17(1), 17-29.

Becerril, H. A., Mao, J., Liu, Z., Stoltenberg, R. M., Bao, Z., \& Chen, Y. (2008). Evaluation of solution-processed reduced graphene oxide films as transparent conductors. ACS nano, 2(3), 463-470.

Burlingame, A. L., Boyd, R. K., \& Gaskell, S. J. (1996). Mass spectrometry. Analytical chemistry, 68(12), 599-652.

Byon, H. R., \& Choi, H. C. (2006). Network single-walled carbon nanotube-field effect transistors (SWNT-FETs) with increased Schottky contact area for highly sensitive biosensor applications. Journal of the American Chemical Society, 128(7), 2188-2189.

Chen, R. J., Zhang, Y., Wang, D., \& Dai, H. (2001). Noncovalent sidewall functionalization of single-walled carbon nanotubes for protein immobilization. Journal of the American Chemical Society, 123(16), 3838-3839.

Collins, P. G., Arnold, M. S., \& Avouris, P. (2001). Engineering carbon nanotubes and nanotube circuits using electrical breakdown. Science, 292(5517), 706-709.

Crowther, J. R. (Ed.). (1995). ELISA: theory and practice (Vol. 42). Springer Science \& Business Media.

Cullen, D. C., Sethi, R. S., \& Lowe, C. R. (1990). Multi-analyte miniature conductance biosensor. Analytica Chimica Acta, 231, 33-40.

Datsyuk, V., Kalyva, M., Papagelis, K., Parthenios, J., Tasis, D., Siokou, A., Kallitsis, I., \& Galiotis, C. (2008). Chemical oxidation of multiwalled carbon nanotubes. Carbon, 46(6), 833840. 
Diamanti-Kandarakis, E., Bourguignon, J. P., Giudice, L. C., Hauser, R., Prins, G. S., Soto, A. M., Zoeller, R. T., \& Gore, A. C. (2009). Endocrine-disrupting chemicals: an Endocrine Society scientific statement. Endocrine reviews, 30(4), 293-342.

Dresselhaus, M. S., Dresselhaus, G., \& Jorio, A. (2004). Unusual properties and structure of carbon nanotubes. Annu. Rev. Mater. Res., 34, 247-278.

Du, D., Huang, X., Cai, J., \& Zhang, A. (2007). Amperometric detection of triazophos pesticide using acetylcholinesterase biosensor based on multiwall carbon nanotube-chitosan matrix. Sensors and Actuators B: Chemical, 127(2), 531-535.

Garcia-Aljaro, C., Cella, L. N., Shirale, D. J., Park, M., Javier Munoz, F., Yates, M. V., \& Mulchandani, A. (2010). Carbon nanotubes-based chemiresistive biosensors for detection of microorganisms. Biosensors and Bioelectronics, 26, 1437-1441.

Geim, A. K., \& Novoselov, K. S. (2007). The rise of graphene. Nature materials, 6(3), 183-191.

Gong, J. L., Sarkar, T., Badhulika, S., \& Mulchandani, A. (2013). Label-free chemiresistive biosensor for mercury (II) based on single-walled carbon nanotubes and structure-switching DNA. Applied physics letters, 102(1), 013701.

Guldi, D. M., Holzinger, M., Hirsch, A., Georgakilas, V., \& Prato, M. (2003). First comparative emission assay of single-wall carbon nanotubes-solutions and dispersions. Chemical Communications, (10), 1130-1131.

Guo, T., Nikolaev, P., Thess, A., Colbert, D. T., \& Smalley, R. E. (1995). Catalytic growth of single-walled manotubes by laser vaporization. Chemical Physics Letters, 243(1), 49-54.

Hamilton, J. C., \& Blakely, J. M. (1980). Carbon segregation to single crystal surfaces of Pt, Pd and Co. Surface Science, 91(1), 199-217.

Härtl, A., Schmich, E., Garrido, J. A., Hernando, J., Catharino, S. C., Walter, S., Feulner, P., Kromka, A., Steinmüller, D., \& Stutzmann, M. (2004). Protein-modified nanocrystalline diamond thin films for biosensor applications. Nature materials, 3(10), 736-742.

He, Q., Wu, S., Yin, Z., \& Zhang, H. (2012). Graphene-based electronic sensors. Chemical Science, 3(6), 1764-1772.

Hernandez, Y., Nicolosi, V., Lotya, M., Blighe, F. M., Sun, Z., De, S., McGovern, I. T., Holland, B., Byrne, M., Gunko, Y. K., Boland, J. J., Niraj, P., Duesberg, G. S., Krishnamurthy, S., Goodhue, R., Hutchison, J., Scardaci, V., Ferrari, A. C., \& Coleman, J. N. (2008). High-yield production of graphene by liquid-phase exfoliation of graphite. Nature nanotechnology, 3(9), $563-568$.

Huang, Y., Dong, X., Liu, Y., Li, L. J., \& Chen, P. (2011). Graphene-based biosensors for detection of bacteria and their metabolic activities. Journal of Materials Chemistry, 21(33), 12358-12362.

Hummers Jr, W. S., \& Offeman, R. E. (1958). Preparation of graphitic oxide. Journal of the American Chemical Society, 80(6), 1339-1339.

Iijima, S. (1991). Helical microtubules of graphitic carbon. Nature, 354(6348), 56-58. 
Jackson, C., Chen, Y. J., \& Mays, J. W. (1996). Size exclusion chromatography with multiple detectors: Solution properties of linear chains of varying flexibility in tetrahydrofuran. Journal of applied polymer science, 61(5), 865-874.

Jiang, X., Ding, W., Luan, C., Ma, Q., \& Guo, Z. (2013). Biosensor for bisphenol A leaching from baby bottles using a glassy carbon electrode modified with DNA and single walled carbon nanotubes. Microchimica Acta, 180(11-12), 1021-1028.

Joshi, K. A., Prouza, M., Kum, M., Wang, J., Tang, J., Haddon, R., Chen, W., \& Mulchandani, A. (2006). V-type nerve agent detection using a carbon nanotube-based amperometric enzyme electrode. Analytical chemistry, 78(1), 331-336.

Kim, S. N., Rusling, J. F., \& Papadimitrakopoulos, F. (2007). Carbon nanotubes for electronic and electrochemical detection of biomolecules. Advanced materials, 19(20), 3214-3228.

Kong, J., Franklin, N. R., Zhou, C., Chapline, M. G., Peng, S., Cho, K., \& Dai, H. (2000). Nanotube molecular wires as chemical sensors. Science, 287(5453), 622-625.

Kong, J., Soh, H. T., Cassell, A. M., Quate, C. F., \& Dai, H. (1998). Synthesis of individual single-walled carbon nanotubes on patterned silicon wafers. Nature, 395(6705), 878-881.

Li, J., Guo, S., Zhai, Y., \& Wang, E. (2009). High-sensitivity determination of lead and cadmium based on the Nafion-graphene composite film. Analytica chimica acta, 649(2), 196-201.

Lian, Y., Yuan, M., \& Zhao, H. (2014). DNA Wrapped Metallic Single-walled Carbon Nanotube Sensor for Pb (II) Detection. Fullerenes, Nanotubes and Carbon Nanostructures, 22(5), 510518.

Lindsey, M. E., Meyer, M., \& Thurman, E. M. (2001). Analysis of trace levels of sulfonamide and tetracycline antimicrobials in groundwater and surface water using solid-phase extraction and liquid chromatography/mass spectrometry. Analytical Chemistry, 73(19), 4640-4646.

Lipscomb, L. D., Vichchulada, P., Bhatt, N. P., Zhang, Q., \& Lay, M. D. (2011). Methods for enhanced control over the density and electrical properties of SWNT networks. Journal of materials science, 46(21), 6812-6822.

Liu, C. W., Huang, C. C., \& Chang, H. T. (2009). Highly selective DNA-based sensor for lead (II) and mercury (II) ions. Analytical chemistry, 81(6), 2383-2387.

Liu, F., Kim, Y. H., Cheon, D. S., \& Seo, T. S. (2013). Micropatterned reduced graphene oxide based field-effect transistor for real-time virus detection. Sensors and Actuators B: Chemical, 186, 252-257.

Liu, N., Cai, X., Lei, Y., Zhang, Q., Chan-Park, M. B., Li, C., Chen, W., \& Mulchandani, A. (2007). Single-Walled Carbon Nanotube Based Real-Time Organophosphate Detector. Electroanalysis, 19(5), 616-619.

Liu, S., \& Guo, X. (2012). Carbon nanomaterials field-effect-transistor-based biosensors. NPG Asia Materials, 4(8), e23.

Liu, Y., \& Wei, W. (2008). Layer-by-layer assembled DNA functionalized single-walled carbon nanotube hybrids for arsenic (III) detection. Electrochemistry Communications, 10(6), 872875. 
Lotya, M., Hernandez, Y., King, P. J., Smith, R. J., Nicolosi, V., Karlsson, L. S., Blighe, F. M., De, S., Wang, Z., McGovern, I. T., Duesberg, G. S., \& Coleman, J. N. (2009). Liquid phase production of graphene by exfoliation of graphite in surfactant/water solutions. Journal of the American Chemical Society, 131(10), 3611-3620.

Maeda, Y., Takano, Y., Sagara, A., Hashimoto, M., Kanda, M., Kimura, S. I., Lian, Y., Nakahodo, T., Tsuchiya, T., Wakahara, T., Akasaka, T., Hasegawa, T., Kazaoui, S., Minami, N., Lu, J., \& Nagase, S. (2008). Simple purification and selective enrichment of metallic SWCNTs produced using the arc-discharge method. Carbon, 46(12), 1563-1569.

Mattevi, C., Kim, H., \& Chhowalla, M. (2011). A review of chemical vapour deposition of graphene on copper. Journal of Materials Chemistry, 21(10), 3324-3334.

McAllister, M. J., Li, J. L., Adamson, D. H., Schniepp, H. C., Abdala, A. A., Liu, J., HerreraAlonso, M., Milius, D. L., Car, R., Prudehomme, R. K., \& Aksay, I. A. (2007). Single sheet functionalized graphene by oxidation and thermal expansion of graphite. Chemistry of Materials, 19(18), 4396-4404.

Morton, J., Havens, N., Mugweru, A., \& Wanekaya, A. K. (2009). Detection of Trace Heavy Metal Ions Using Carbon Nanotube-Modified Electrodes. Electroanalysis, 21(14), 1597-1603.

Mulchandani, A., Chen, W., Mulchandani, P., Wang, J., \& Rogers, K. R. (2001). Biosensors for direct determination of organophosphate pesticides. Biosensors and bioelectronics, 16(4), 225-230.

Novoselov, K. S., Geim, A. K., Morozov, S. V., Jiang, D., Zhang, Y., Dubonos, S. V., Grigorieva, I. V., \& Firsov, A. A. (2004). Electric field effect in atomically thin carbon films. Science, 306(5696), 666-669.

Obraztsov, A. N., Obraztsova, E. A., Tyurnina, A. V., \& Zolotukhin, A. A. (2007). Chemical vapor deposition of thin graphite films of nanometer thickness. Carbon, 45(10), 2017-2021.

Ohno, Y., Maehashi, K., \& Matsumoto, K. (2010). Label-free biosensors based on aptamermodified graphene field-effect transistors. Journal of the American Chemical Society, 132(51), 18012-18013.

Odom, T. W., Huang, J. L., Kim, P., \& Lieber, C. M. (1998). Atomic structure and electronic properties of single-walled carbon nanotubes. Nature, 391(6662), 62-64.

Oliveira, S. C. B., Corduneanu, O., \& Oliveira-Brett, A. M. (2008). In situ evaluation of heavy metal-DNA interactions using an electrochemical DNA biosensor. Bioelectrochemistry, 72(1), 53-58.

Paredes, J. I., Villar-Rodil, S., Martinez-Alonso, A., \& Tascon, J. M. D. (2008). Graphene oxide dispersions in organic solvents. Langmuir, 24(19), 10560-10564.

Park, M., Cella, L. N., Chen, W., Myung, N. V., \& Mulchandani, A. (2010). Carbon nanotubesbased chemiresistive immunosensor for small molecules: Detection of nitroaromatic explosives. Biosensors and Bioelectronics, 26(4), 1297-1301.

Pedrosa, V. A., Paliwal, S., Balasubramanian, S., Nepal, D., Davis, V., Wild, J., Ramanculov, E., \& Simonian, A. (2010). Enhanced stability of enzyme organophosphate hydrolase interfaced on the carbon nanotubes. Colloids and Surfaces B: Biointerfaces, 77(1), 69-74. 
Pennington, J. C., \& Brannon, J. M. (2002). Environmental fate of explosives. Thermochimica Acta, 384(1), 163-172.

Periyakaruppan, A., Arumugam, P. U., Meyyappan, M., \& Koehne, J. E. (2011). Detection of ricin using a carbon nanofiber based biosensor. Biosensors and Bioelectronics, 28(1), 428433.

Pumera, M., Ambrosi, A., Bonanni, A., Chng, E. L. K., \& Poh, H. L. (2010). Graphene for electrochemical sensing and biosensing. TrAC Trends in Analytical Chemistry, 29(9), 954965.

Rodríguez-Lázaro, D., D'Agostino, M., Herrewegh, A., Pla, M., Cook, N., \& Ikonomopoulos, J. (2005). Real-time PCR-based methods for detection of Mycobacterium avium subsp. paratuberculosis in water and milk. International journal of food microbiology, 101(1), 93104.

Sarkar, T., Gao, Y., \& Mulchandani, A. (2013). Carbon Nanotubes-Based Label-Free Affinity Sensors for Environmental Monitoring. Applied biochemistry and biotechnology, 170(5), 1011-1025.

Scheibe, B., Rümmeli, M. H., Borowiak-Palen, E., \& Kalenczuk, R. J. (2011). Separation of surfactant functionalized single-walled carbon nanotubes via free solution electrophoresis method. Central European Journal of Physics, 9(2), 325-329.

Shao, Y., Wang, J., Wu, H., Liu, J., Aksay, I. A., \& Lin, Y. (2010). Graphene based electrochemical sensors and biosensors: a review. Electroanalysis, 22(10), 1027-1036.

Sherma, J. (1993). Pesticides. Analytical chemistry, 65(12), 40R-54R.

So, H. M., Park, D. W., Jeon, E. K., Kim, Y. H., Kim, B. S., Lee, C. K., Choi, S. Y., Kim, S. C., Chang, H., \& Lee, J. O. (2008). Detection and Titer Estimation of Escherichia coli Using Aptamer-Functionalized Single-Walled Carbon-Nanotube Field-Effect Transistors. Small, 4(2), 197-201.

Srivastava, A., Galande, C., Ci, L., Song, L., Rai, C., Jariwala, D., Kelly, K. F., \& Ajayan, P. M. (2010). Novel liquid precursor-based facile synthesis of large-area continuous, single, and few-layer graphene films. Chemistry of Materials, 22(11), 3457-3461.

Stankovich, S., Dikin, D. A., Piner, R. D., Kohlhaas, K. A., Kleinhammes, A., Jia, Y., Wu, Y., Nguyen, S. T., \& Ruoff, R. S. (2007). Synthesis of graphene-based nanosheets via chemical reduction of exfoliated graphite oxide. Carbon, 45(7), 1558-1565.

Steinitz, M. (2000). Quantitation of the blocking effect of Tween 20 and bovine serum albumin in ELISA microwells. Analytical biochemistry, 282(2), 232-238.

Tanimoto de Albuquerque, Y. D., \& Ferreira, L. F. (2007). Amperometric biosensing of carbamate and organophosphate pesticides utilizing screen-printed tyrosinase-modified electrodes. Analytica chimica acta, 596(2), 210-221.

Thévenot, D. R., Toth, K., Durst, R. A., \& Wilson, G. S. (2001). Electrochemical biosensors: recommended definitions and classification. Biosensors and Bioelectronics, 16(1), 121-131. 
Tlili, C., Cella, L. N., Myung, N. V., Shetty, V., \& Mulchandani, A. (2010). Single-walled carbon nanotube chemoresistive label-free immunosensor for salivary stress biomarkers. Analyst, 135(10), 2637-2642.

Van Loon, J. A. (2012). Analytical atomic absorption spectroscopy: selected methods. Elsevier.

Wanekaya, A. K., Chen, W., \& Mulchandani, A. (2008). Recent biosensing developments in environmental security. Journal of Environmental Monitoring, 10(6), 703-712.

Wang, J. (2005). Carbon-nanotube based electrochemical biosensors: A review. Electroanalysis, 17(1), 7-14.

Wang, J., Musameh, M., \& Lin, Y. (2003). Solubilization of carbon nanotubes by Nafion toward the preparation of amperometric biosensors. Journal of the American Chemical Society, 125(9), 2408-2409.

Wang, J., Yang, S., Guo, D., Yu, P., Li, D., Ye, J., \& Mao, L. (2009). Comparative studies on electrochemical activity of graphene nanosheets and carbon nanotubes. Electrochemistry Communications, 11(10), 1892-1895.

Wilder, J. W., Venema, L. C., Rinzler, A. G., Smalley, R. E., \& Dekker, C. (1998). Electronic structure of atomically resolved carbon nanotubes. Nature, 391(6662), 59-62.

Xu, K., Meshik, X., Nichols, B. M., Zakar, E., Dutta, M., \& Stroscio, M. A. (2014). Grapheneand aptamer-based electrochemical biosensor. Nanotechnology, 25(20), 205501.

Y1lmaz, F., Özdemir, N., Demirak, A., \& Tuna, A. L. (2007). Heavy metal levels in two fish species Leuciscus cephalus and Lepomis gibbosus. Food Chemistry, 100(2), 830-835.

Zelada-Guillén, G. A., Riu, J., Düzgün, A., \& Rius, F. X. (2009). Immediate detection of living bacteria at ultralow concentrations using a carbon nanotube based potentiometric aptasensor. Angewandte Chemie International Edition, 48(40), 7334-7337.

Zheng, M., Jagota, A., Strano, M. S., Santos, A. P., Barone, P., Chou, S. G., Diner, B. A., Dresselhaus, M. S., Mclean, R. S., Onoa, G. B., Samsonidze, G. G., Semke, E. D., Usrey, M., \& Walls, D. J. (2003). Structure-based carbon nanotube sorting by sequence-dependent DNA assembly. Science, 302(5650), 1545-1548.

Zhou, L., Li, D. J., Gai, L., Wang, J. P., \& Li, Y. B. (2012). Electrochemical aptasensor for the detection of tetracycline with multi-walled carbon nanotubes amplification. Sensors and Actuators B: Chemical, 162(1), 201-208.

Zhou, M., Wang, Y., Zhai, Y., Zhai, J., Ren, W., Wang, F., \& Dong, S. (2009). Controlled Synthesis of Large-Area and Patterned Electrochemically Reduced Graphene Oxide Films. Chemistry-A European Journal, 15(25), 6116-6120.

Zhou, M., Zhai, Y., \& Dong, S. (2009). Electrochemical sensing and biosensing platform based on chemically reduced graphene oxide. Analytical Chemistry, 81(14), 5603-5613.

Zi-Pu, H., Ogletree, D. F., Van Hove, M. A., \& Somorjai, G. A. (1987). LEED theory for incommensurate overlayers: Application to graphite on Pt (111). Surface Science, 180(2), 433459. 
Fig. 1 (a): http://www.nature.com/nmat/journal/v6/n3/images/nmat1849-f1.jpg

802 Fig. 1 (b): http://www.nature.com/nmat/journal/v6/n3/images/nmat1849-f3.jpg

803

(a)
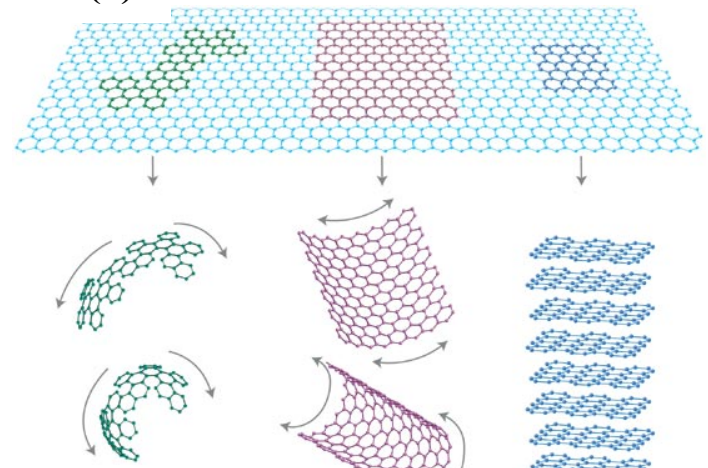

804

805

806

807

808
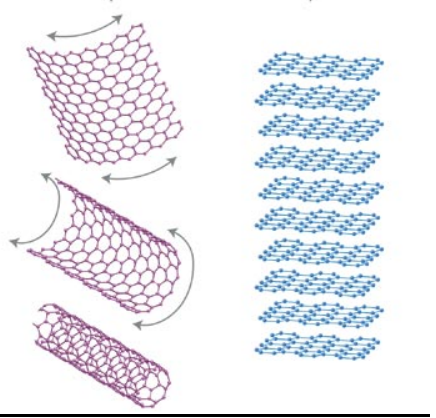
monolayer graphene (Geim and Novoselov, 2007)

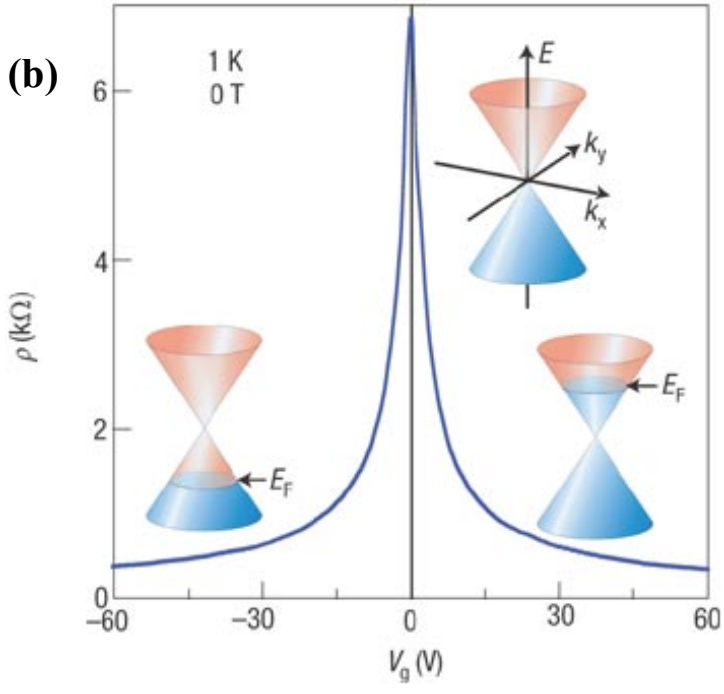

홍
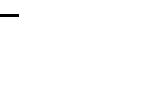

Fig. 1 (a) Graphene: building block for all other carbon allotropes, (b) ambipolar electric field effects in 


\section{Fig. 2:}

810 http://pubs.rsc.org/services/images/RSCpubs.ePlatform.Service.FreeContent.ImageService.svc/ImageSe 811 rvice/Articleimage/2011/JM/c0jm02126a/c0jm02126a-f6.gif

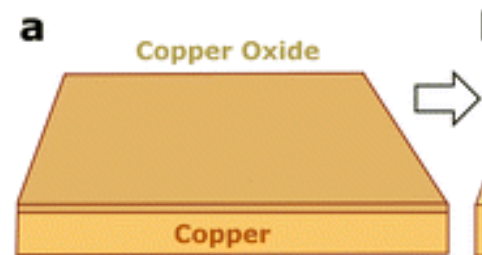
b $1000 \mathrm{C}, \mathrm{CH}_{4} / \mathrm{H}_{2} \quad$ C

814 Fig. 2 Schematic showing evolution of graphene growth via CVD on a $\mathrm{Cu}$ foil: (a) annealing of $\mathrm{Cu}$ foil 815 and enlargement of $\mathrm{Cu}$ grains, (b) decomposition of hydrocarbons resulting in nucleation of graphene 816 grains, (c) growth of graphene from the nucleation sites (Mattevi et al., 2011). 
818 Fig. 3: http://www.nature.com/nature/journal/v391/n6662/images/391059aa.tif.2.gif

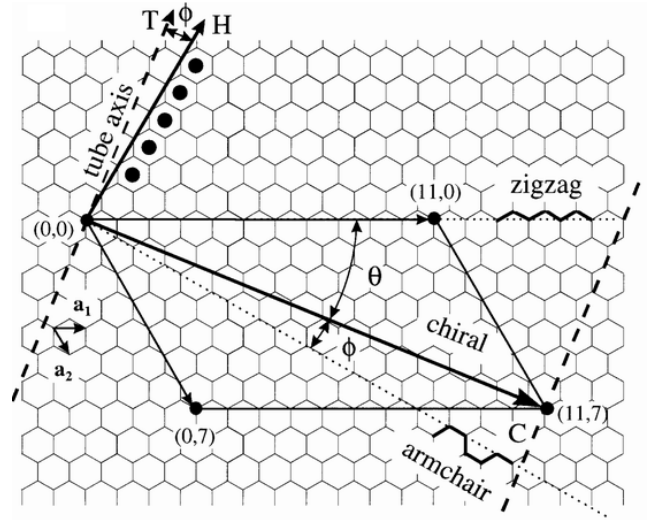

Fig. 3 Construction of carbon nanotube from a single graphene sheet by rolling up sheet along wrapping vector C. Wrapping vectors along the dotted lines lead to tubes that are zigzag or armchair (Wilder et al., 823 1998). 
825 Fig. 4:

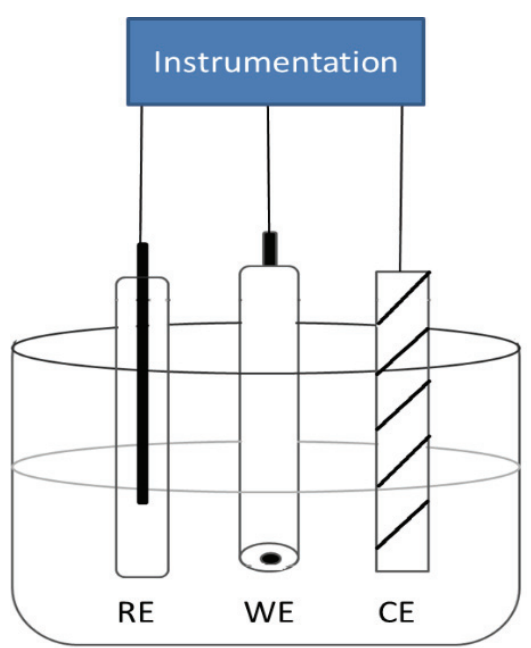

826

827

828 Fig. 4 Schematic representation of a three electrode system

829 

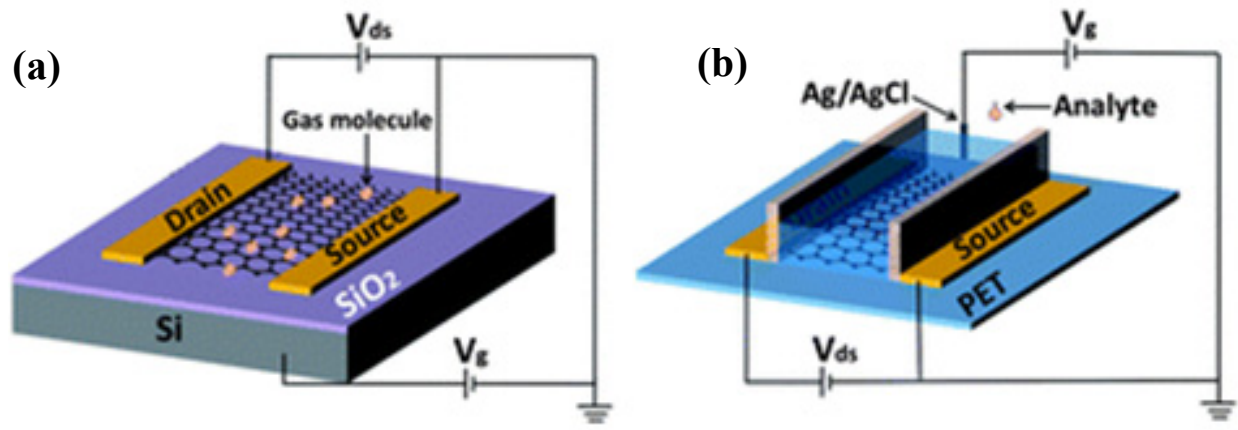

836

(c)

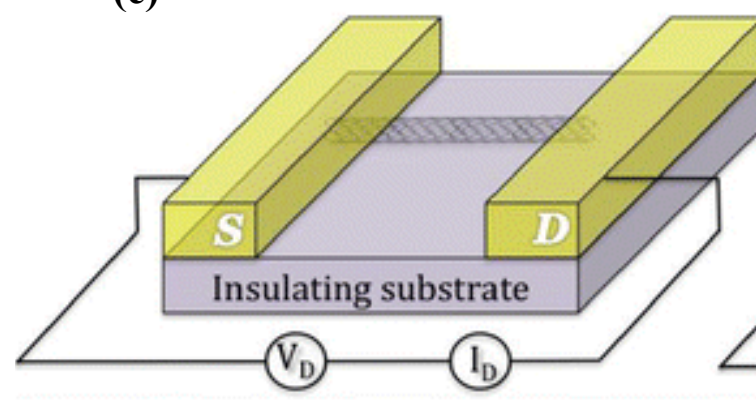

(d)

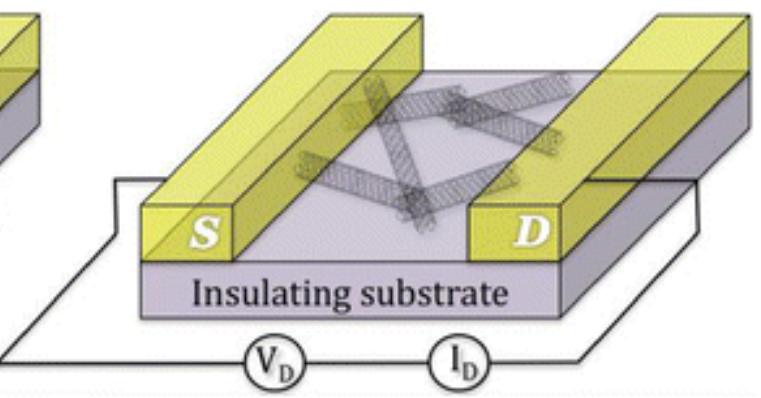

Fig. 5 (a) Back-gated graphene-FET on $\mathrm{Si} / \mathrm{SiO} 2$ substrate, (b) solution-gated graphene-FET on a flexible polyethylene terephthalate (PET) substrate (He et al., 2012). SWNT-based chemiresistor configuration showing (c) single SWNT and (d) network of SWNTs as conducting channel (Kim et al., 2007). 
843

844

845

846

847

848 Fig. 6 Irreversible adsorption of pyrene moiety onto sidewall of a SWNT via $\pi$ stacking (Chen et al., 849 2001).

Fig. 6:

http://pubs.acs.org/appl/literatum/publisher/achs/journals/content/jacsat/2001/jacsat.2001.12

3.issue-16/ja010172b/production/images/medium/ja010172bh00001.gif
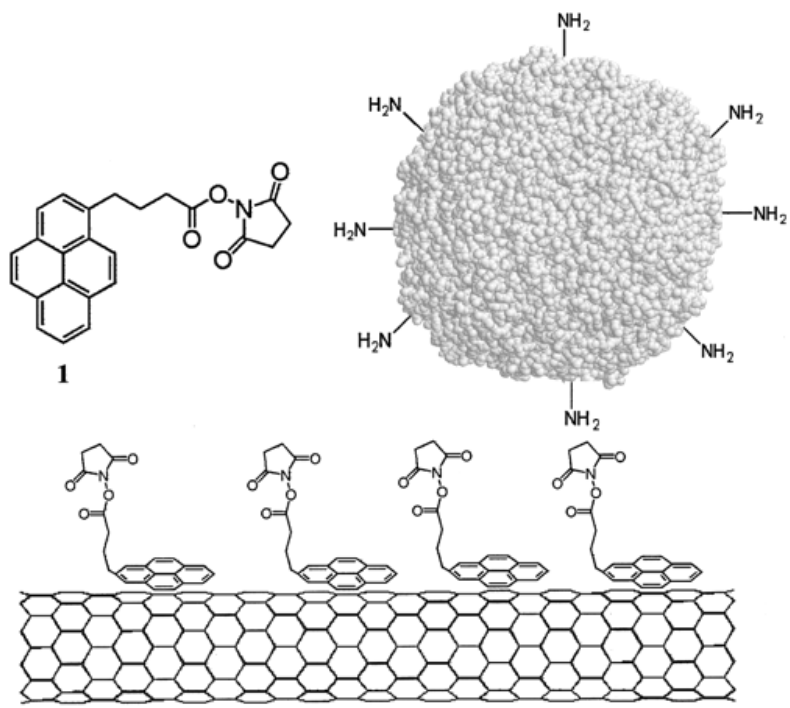

850 
Fig. 7: http://ars.els-cdn.com/content/image/1-s2.0-S0925400513006801-gr2.jpg

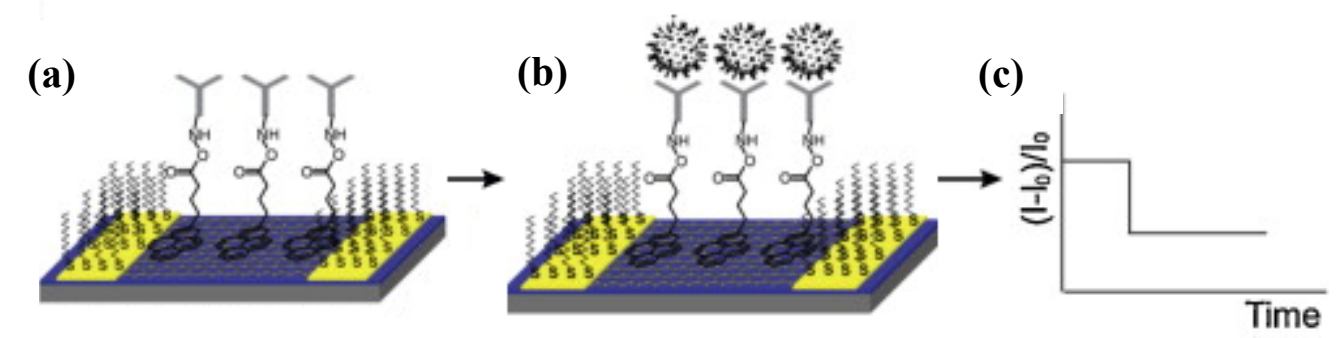

853

854

855 Fig. 7 Schematic of reduced graphene oxide - FET based rotavirus detection: (a) antibody 856 immobilization, (b) rotavirus capture, (c) electrical signal detection (Liu et al., 2013). 
Fig. 8:

861 http://onlinelibrary.wiley.com/store/10.1002/anie.200902090/asset/image n/nfig002.gif?v=1\& $\mathrm{t}=\mathrm{i} 8 \mathrm{j} 84 \mathrm{~b} 9 \mathrm{f} \& \mathrm{~s}=\mathrm{bf} 418 \mathrm{~d} 1 \mathrm{a} 284186 \mathrm{be38c4abf7a5b0e337567ef76b}$

(a)

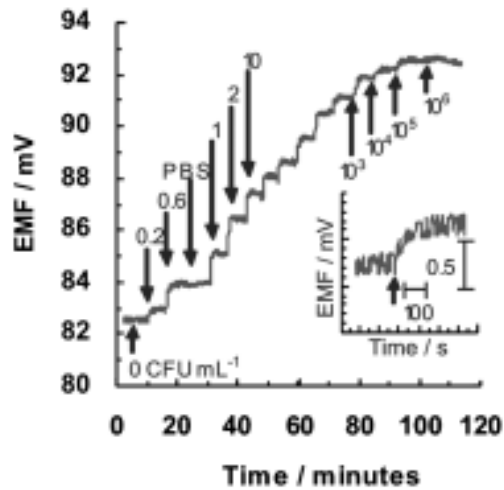

862

863

864

865

866 corresponding calibration curve (Zelada-Guillén et al., 2009). (b)

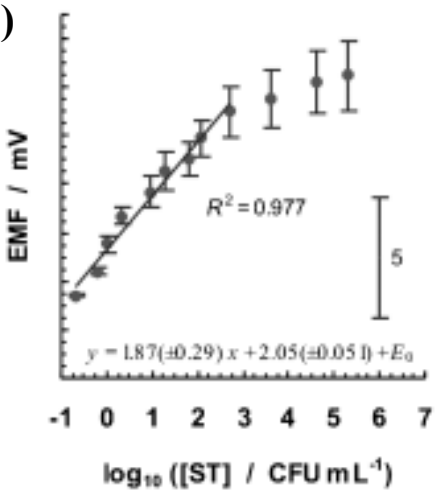

Fig. 8(a) Potentiometric response of sensor against stepwise increase of bacteria concentration, (b) 
Fig. 9(a):

Fig. 9(b,c):

(a)

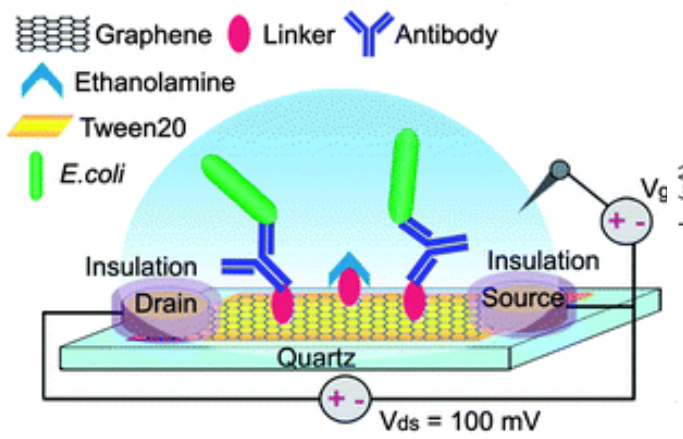

(b)

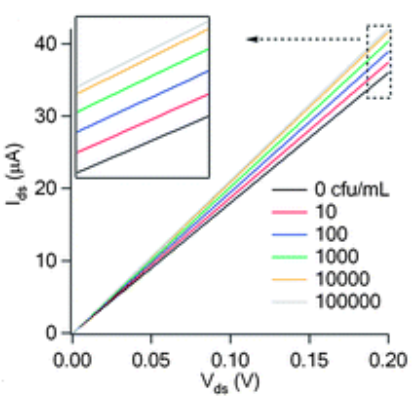

(c)

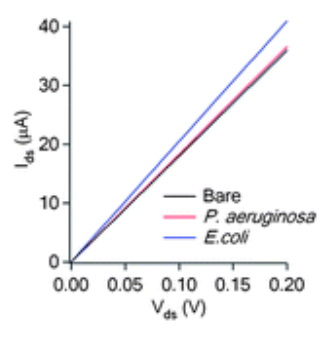

876 Fig. 9 (a) Illustration of anti-E. coli antibody functionalized graphene-FET for detection of E. coli, (b) $877 \mathrm{I}_{\mathrm{ds}}-\mathrm{V}_{\mathrm{ds}}$ curves of anti-E. coli antibody functionalized graphene device after incubated with $E$. coli of 878 different concentrations $\left(\mathrm{V}_{\mathrm{g}}=0 \mathrm{~V}\right)$, (c) $\mathrm{I}_{\mathrm{ds}}-\mathrm{V}_{\mathrm{ds}}$ curves of antibody functionalized graphene device before 879 and after incubated with $P$. aeruginosa and E. coli (both $\left.10^{5} \mathrm{cfu} / \mathrm{mL}\right)\left(\mathrm{V}_{\mathrm{g}}=0 \mathrm{~V}\right)$ (Huang et al., 2011). 
Fig. 10(a): http://pubs.rsc.org/services/images/RSCpubs.ePlatform.Service.FreeContent.ImageService.svc/I mageService/Articleimage/2011/JM/c1jm11436k/c1jm11436k-f5.gif http://pubs.rsc.org/services/images/RSCpubs.ePlatform.Service.FreeContent.ImageService.svc/I mageService/Articleimage/2011/JM/c1jm11436k/c1jm11436k-f6.gif
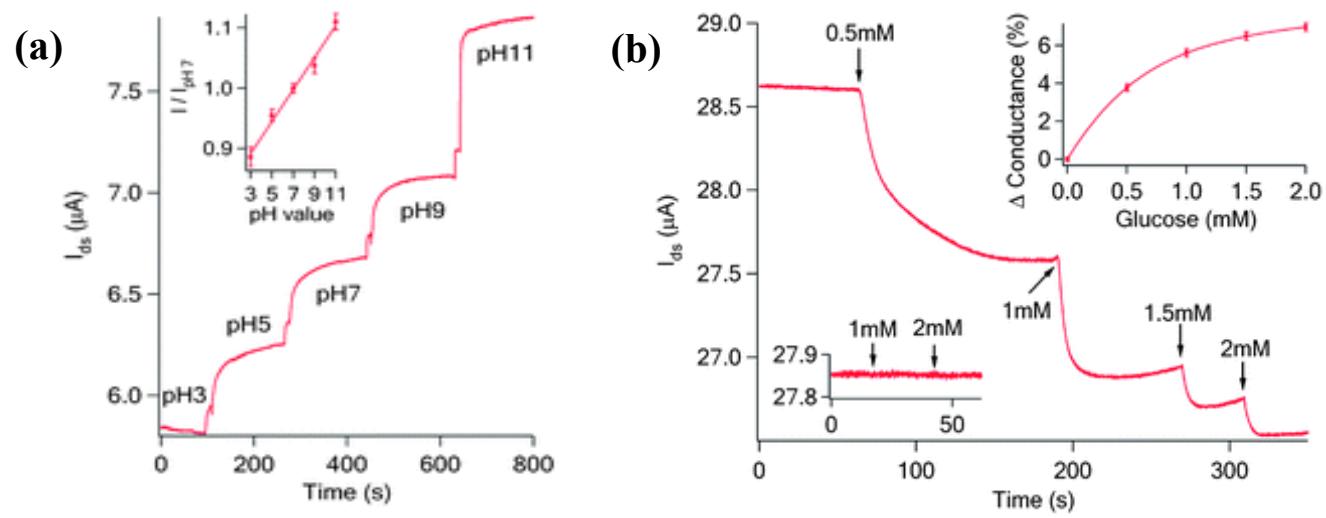

Fig. 10 (a) Real-time current of graphene device for different $\mathrm{pH}$ solutions $\left(\mathrm{V}_{\mathrm{ds}}=100 \mathrm{mV}, \mathrm{V}_{\mathrm{g}}=0 \mathrm{~V}\right)$, (b) Real-time current recording $\left(\mathrm{V}_{\mathrm{ds}}=100 \mathrm{mV}\right.$ and $\left.\mathrm{V}_{\mathrm{g}}=0 \mathrm{~V}\right)$ of graphene device immobilized with E. coli bacteria with application of glucose at the indicated time points; Lower inset: bacteria-free graphene sensor was not responsive to glucose. Upper inset: Percentage change in graphene conductance versus

893 glucose concentration (Huang et al., 2011). 

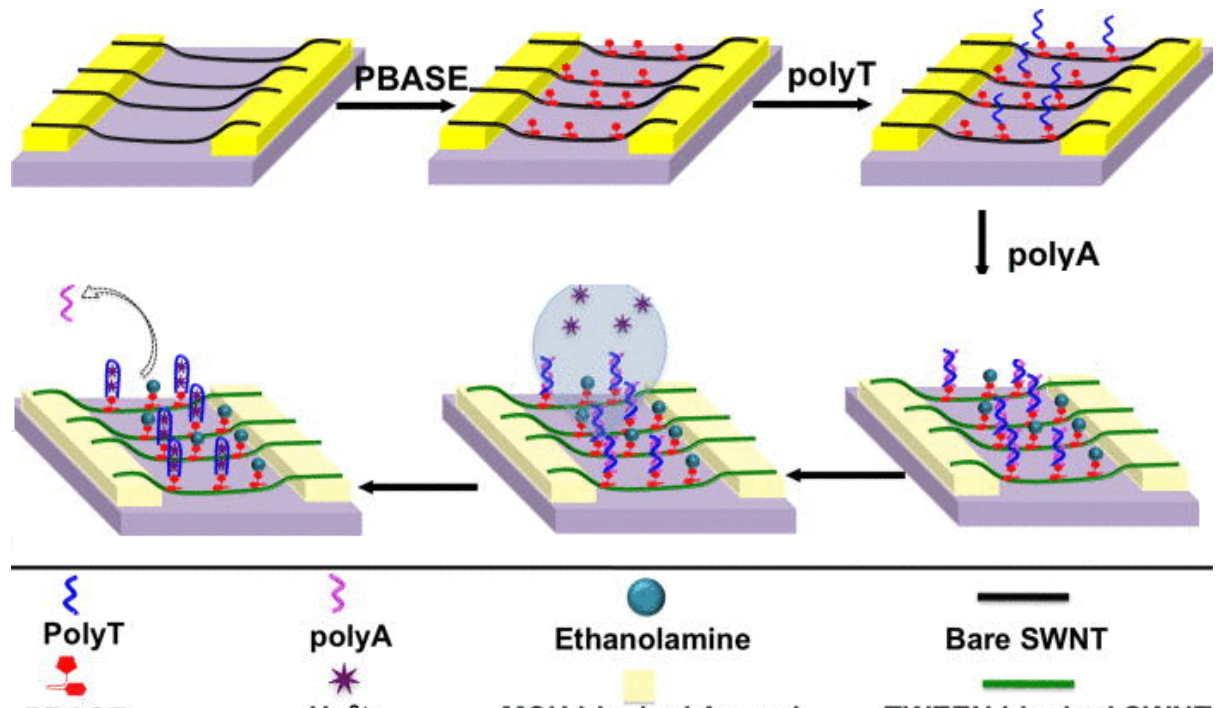
polyA PBASE
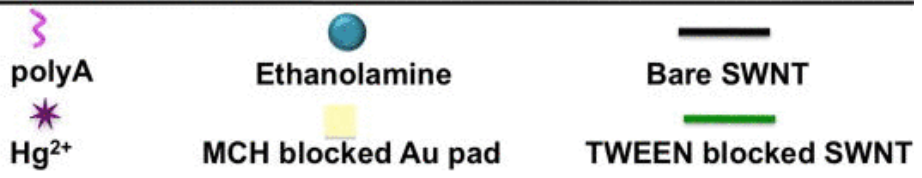

898

899 Fig. 11 Schematic illustration of SWNT chemiresistive label-free biosensor fabrication via covalent 900 functionalization with amino-labeled polyT followed by hybridization with polyA (Gong et al., 2013). 
902

903

904

905

906

907

908

909

910

911

Fig. 12(a): http://scitation.aip.org/docserver/fulltext/aip/journal/apl/102/1/1.4773569.online.f3.gif

Fig. 12(b): http://scitation.aip.org/docserver/fulltext/aip/journal/apl/102/1/1.4773569.online.f4.gif

(a)

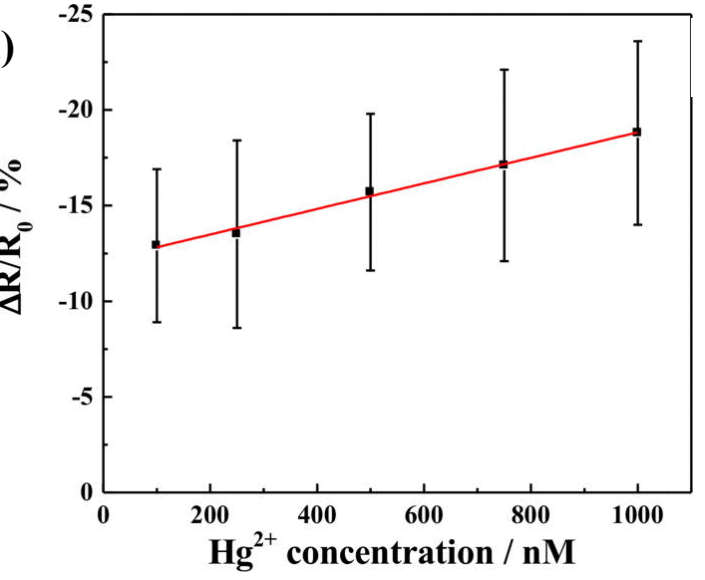

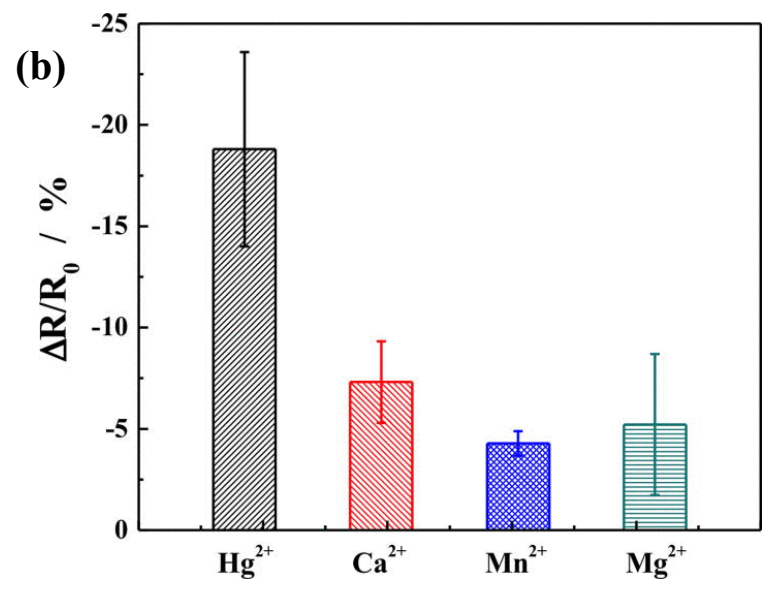

Fig. 12 (a) SWNT chemiresistive label-free biosensor calibration for $\mathrm{Hg}^{2+}$ concentrations varying from $100 \mathrm{nM}$ to $1 \mu \mathrm{M}$; (b) Responses of SWNT chemiresistive label-free biosensor for different metal ions. The concentrations of the metal ions were $1 \mu \mathrm{M}$ (Gong et al., 2013). 
Fig. 13:
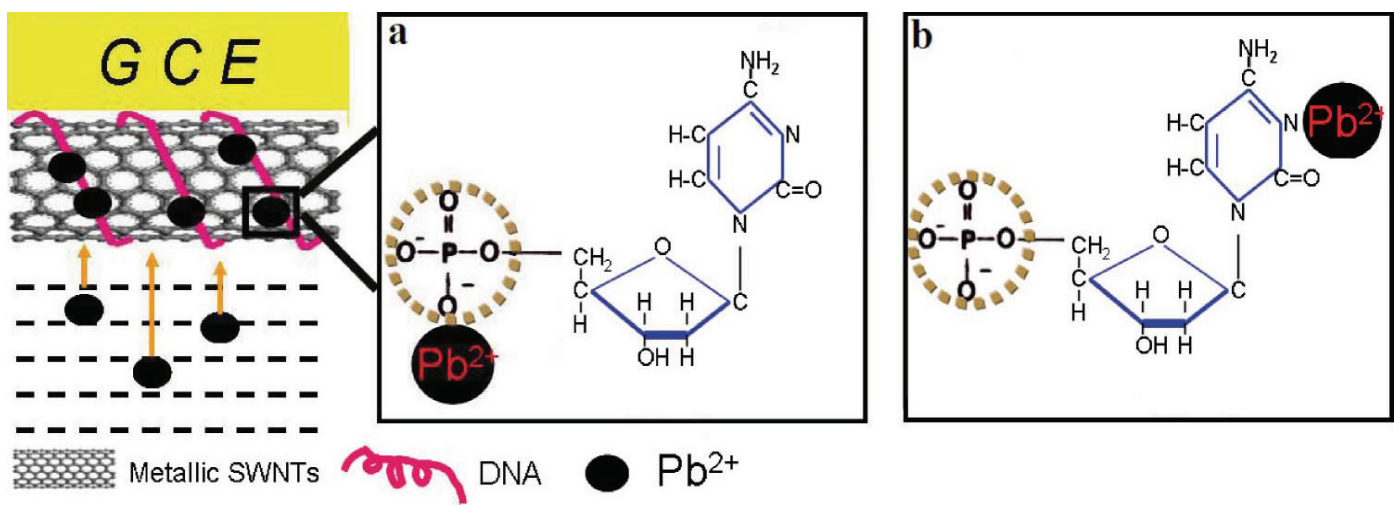

913

914

915 Fig. 13 Schematic diagram of $\mathrm{Pb}^{2+}$ detection on met-SWNTs/DNA/GCE surface due to interactions 916 between $\mathrm{Pb} 2+$ and phosphate groups (a) or bases of DNA fragments (b) (Lian et al., 2014). 
Fig. 14: http://ars.els-cdn.com/content/image/1-s2.0-S0925400507003322-gr3.jpg

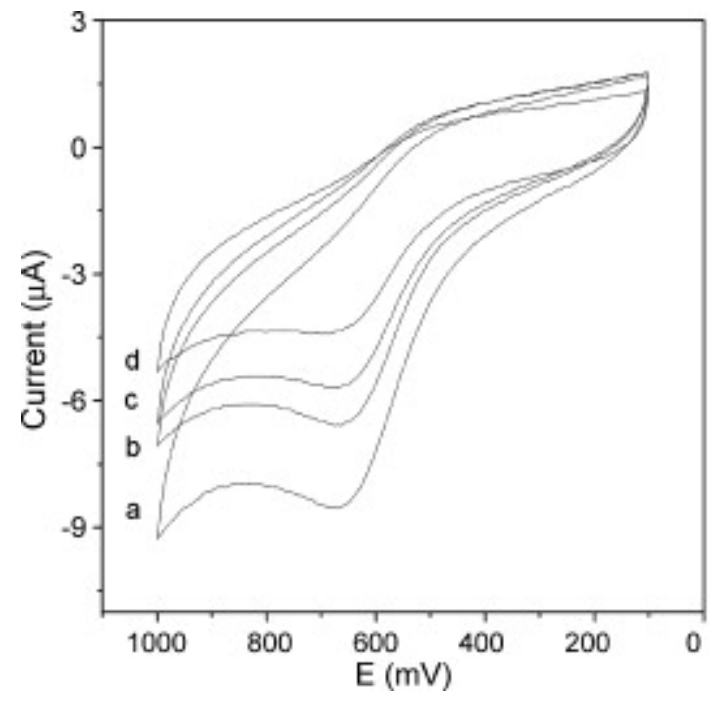

920

921 Fig. $14 \mathrm{CVs}$ of AChE immobilized MWNT electrodes in $\mathrm{pH} 7.0$ PBS containing $0.4 \mathrm{mM}$ 922 acetylthiocholine after incubation in $0 \mu \mathrm{M}(\mathrm{a}), 1.5 \mu \mathrm{M}(\mathrm{b}), 3.5 \mu \mathrm{M}$ (c) and $5.2 \mu \mathrm{M}$ (d) triazophos solution 923 for $10 \mathrm{~min}$. (Du et al., 2007)

924 
Fig. 15: http://ars.els-cdn.com/content/image/1-s2.0-S0927776510000378-gr5.jpg
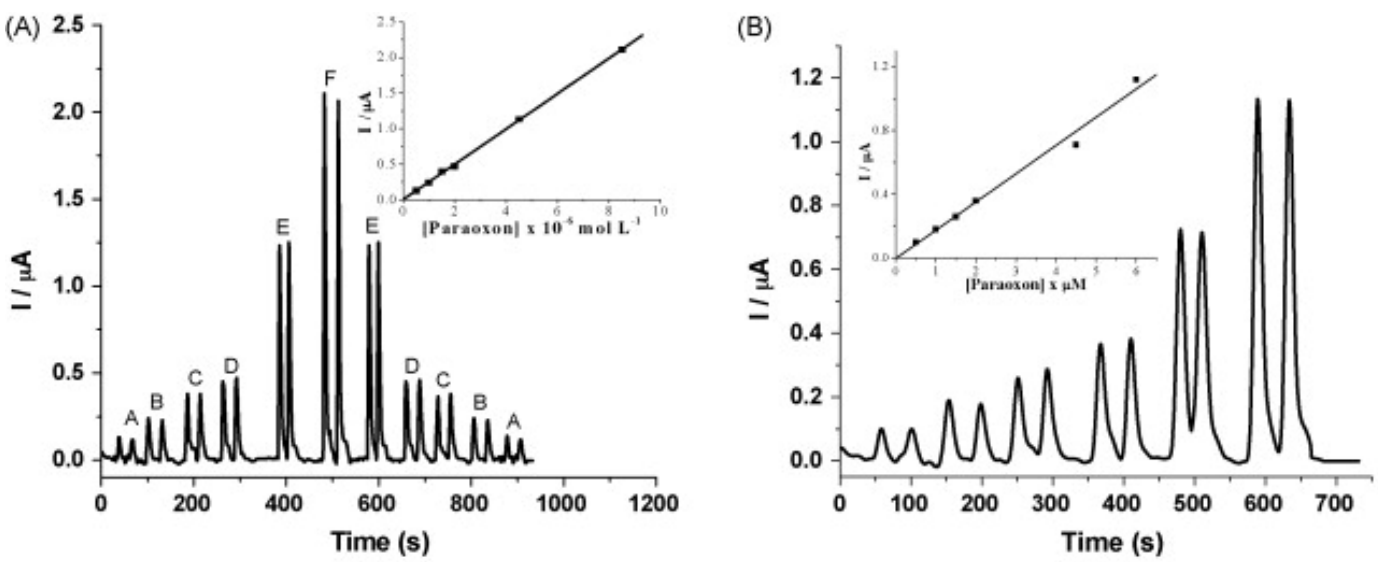

Fig. 15 Flow-injection on (A) OPH-SWNTs electrode and (B) OPH-MWNTs electrode with sequential 929 injection of paraoxon (A) 0.5, (B) 1.0, (C) 1.5, (D) 2.0, (E) 4.5 and (F) $8.0 \mu \mathrm{M}$ at applied potential of $930 \quad 0.8 \mathrm{~V}$ vs $\mathrm{Ag} / \mathrm{AgCl}$ and flow rate $1 \mathrm{~mL} / \mathrm{s}$ (Pedrosa et al., 2010)

931 
931

932

933

934

935

936 Fig. 16 (a) DPVs of different concentrations of BPA in pH 8.5 phosphate buffer solution, (b) 937 corresponding calibration curve (Jiang et al., 2013).
Fig. 16: http://static-content.springer.com/image/art\%3A10.1007\%2Fs00604-013-10254/MediaObjects/604 20131025 Figa HTML.gif

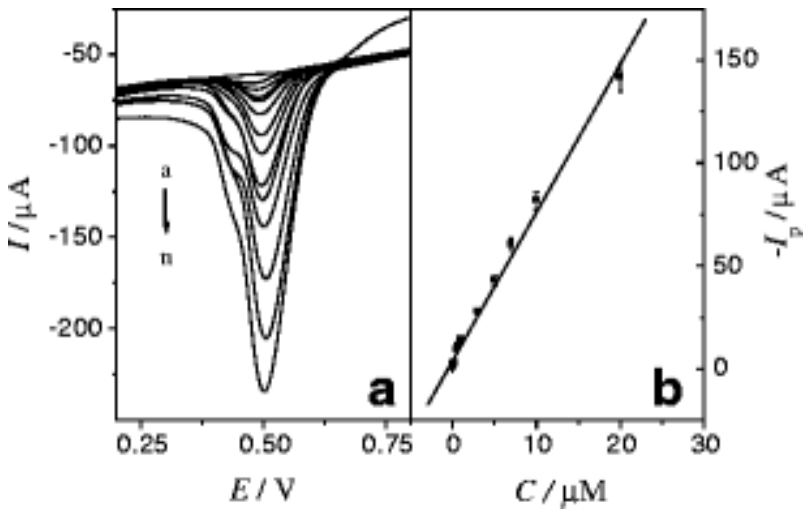

938 


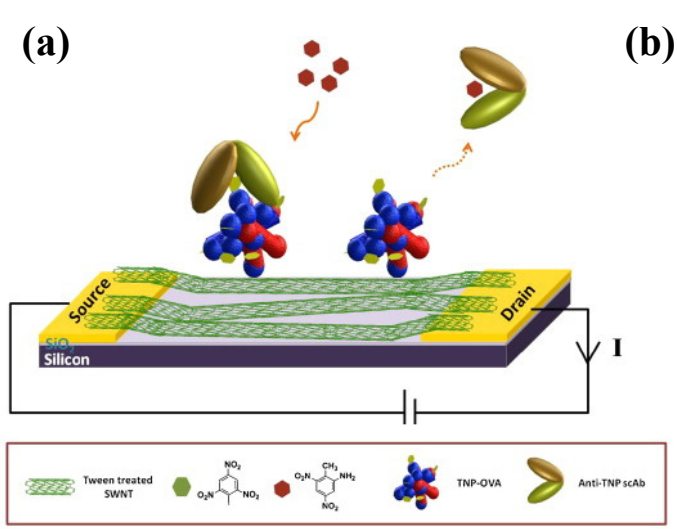

(b)

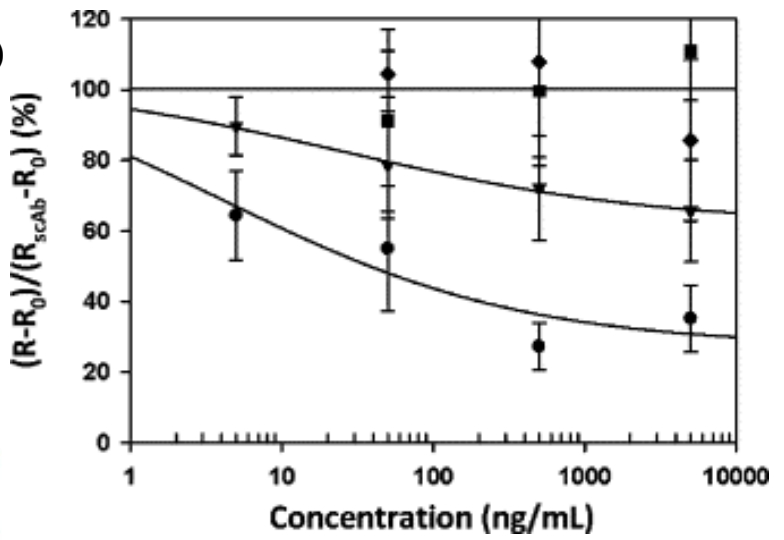

942

943

944 Fig. 17 (a) Schematic diagram of the SWNT-based immunosensor to detect TNT. (b) Calibration curves 945 of the sensor for different derivatives of TNT: TNB $(\bullet), 2 \mathrm{~A}-4,6-\mathrm{DNT}(\boldsymbol{\nabla}), 2,4-\mathrm{DNT}(\bullet)$, and toluene ( $\boldsymbol{\bullet})$ 946 (Park et al., 2010).

947 
Fig. 18(a): http://ars.els-cdn.com/content/image/1-s2.0-S0925400511011579-gr2.jpg

Fig. 18(b): http://ars.els-cdn.com/content/image/1-s2.0-S0925400511011579-gr7.jpg

Fig. 18(c): http://ars.els-cdn.com/content/image/1-s2.0-S0925400511011579-gr8.jpg

(a)
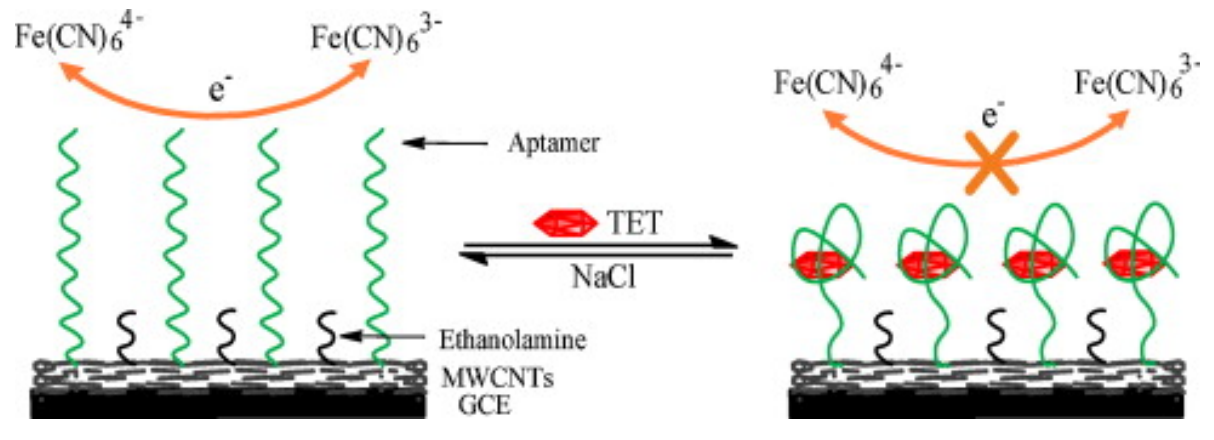

952

953

(b)

(c)
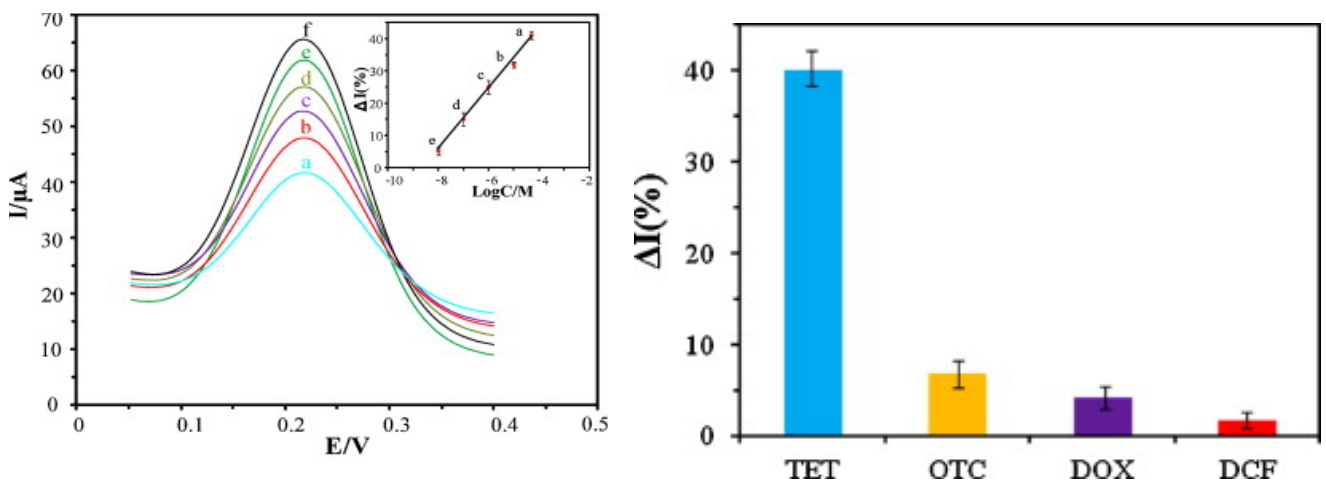

955

956

Fig. 18 (a) Mechanism of electrochemical aptamer-based sensor for detection of tetracycline, (b) DPV of the aptasensor incubated with different concentrations of TET. Inset shows the linear relationship between the anodic peak current changes versus log of TET concentrations. (c) Specificity of the aptasensor to 50 $\mu \mathrm{M}$ TET by comparing it to the interfering agents, including two structurally similar tetracycline 
964 Table 1: List of recent reports in the literature on carbon nanomaterials based electrochemical 965 biosensors for detection of pathogens and heavy metals

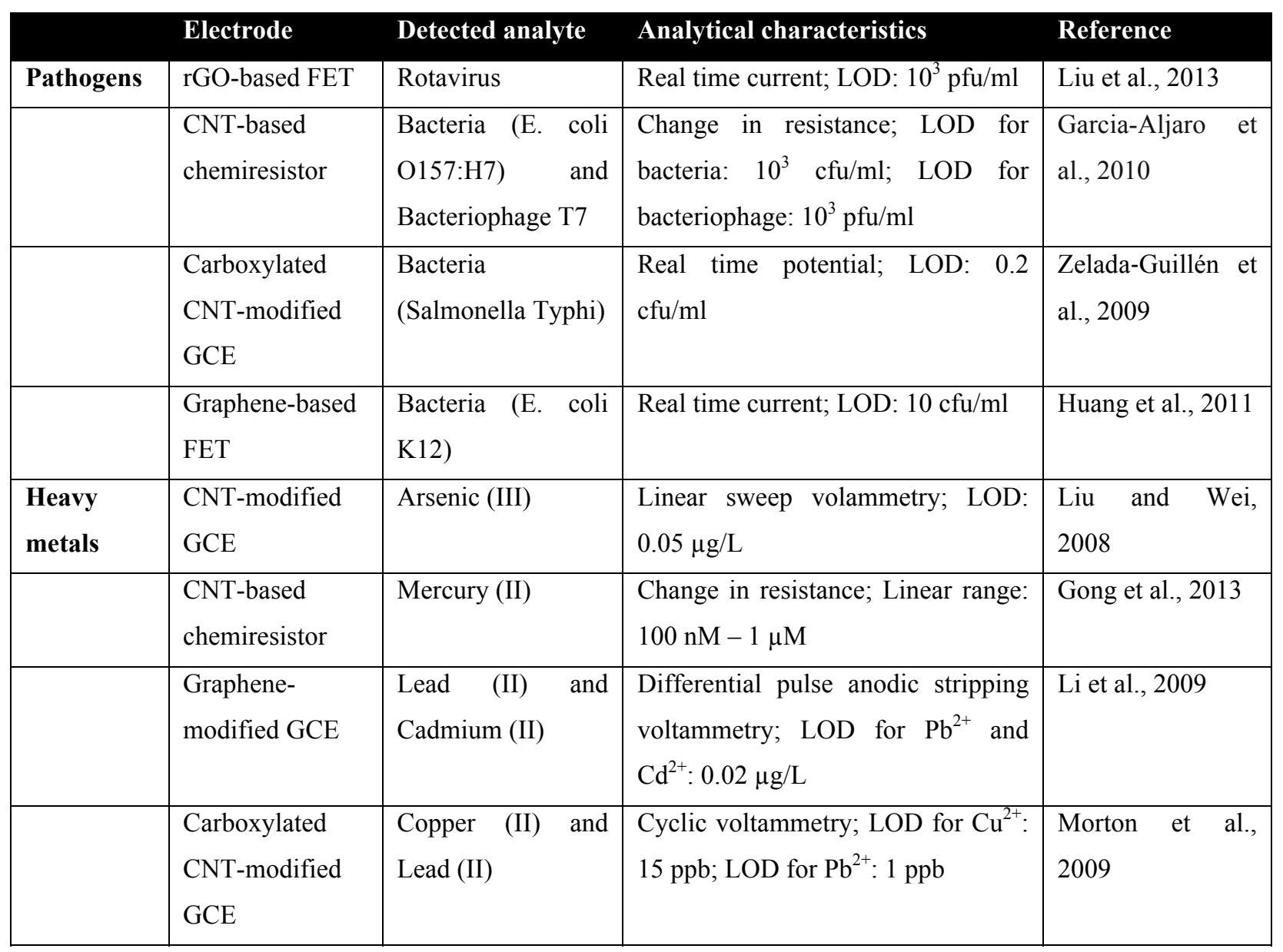

